UNIVERSIDADE DE SÃO PAULO INSTITUTO DE GEOCIÊNCIAS

\title{
PALEOBIOLOGIA DE FORAMINÍFEROS \\ E MICROFÓSSEIS ASSOCIADOS DOS DEPÓSITOS \\ EOCÊNICOS, MIOCÊNICOS E PLIO-PLEISTOCÊNICOS \\ DA ILHA SEYMOUR, ANTÁRTICA OCIDENTAL
}

\author{
VICTOR CEZAR SOFICIER BADARÓ
}

ORIENTADOR: PROF. DR. SETEMBRINO PETRI

TESE DE DOUTORADO

$\mathrm{N}^{\circ} 576$

COMISSÃO JULGADORA

DR. SETEMBRINO PETRI

DR. THOMAS RICH FAIRCHILD

Dra. Silvia Helena de Mello e Souza

DRA. KAREN BADARACO COSTA

DRA. CÁtIA FERnANDES BARbOSA

SÃo PAULO

2017 
BADARÓ, VICTOR CEZAR SOFICIER BADARÓ

PALEOBIOLOGIA DE FORAMINÍFEROS E MICROFÓSSEIS ASSOCIADOS DOS DEPÓSITOS EOCÊNICOS, MIOCÊNICOS E PLIO-PLEISTOCÊNICOS DA ILHA SEYMOUR, ANTÁRTICA OCIDENTAL / VICTOR CEZAR SOFICIER BADARÓ - SÃO PAULO, 2017

310 P.: IL + 1 ANEXO

TESE (DOUTORADO) : IGC-USP

ORIENTADOR: PETRI, SETEMBRINO

1. Paleobiologia 2. Microfósseis 3. Foraminíferos

I. TÍTULO 
"A maior parte do que sabemos é a menor do que ignoramos." Padre António Vieira, Sete propriedades da alma aplicadas às obrigações de um válido (16--)

\begin{abstract}
"There is nothing like geology; the pleasure of the first day's partridge shooting or first day's hunting cannot be compared to finding a fine group of fossil bones, which tell their story of former times with almost a living tongue."

Charles Robert Darwin, em carta para sua irmã Catherine (6 de abril de 1834)
\end{abstract}

“(...) the solemn duty of all true curators: to preserve the remains when we cannot rescue the living, and to maintain the records when we cannot even conserve the remains - lest we forget, lest we forget (...)" Stephen Jay Gould, The dodo in the caucus cace (1996)

"The many men, so beautiful!

And they all dead did lie: And a thousand thousand slimy things Lived on; and so did I.

O happy living things! no tongue

Their beauty might declare: A spring of love gushed from my heart, And I blessed them unaware: Sure my kind saint took pity on me, And I blessed them unaware.

The self-same moment I could pray; And from my neck so free The Albatross fell off, and sank Like lead into the sea." Samuel Taylor Coleridge, The rime of the ancient mariner (1834) 



\section{SUMÁRIO}

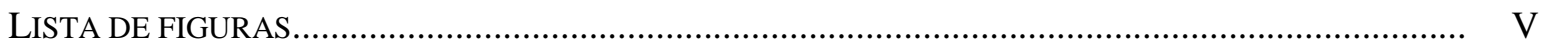

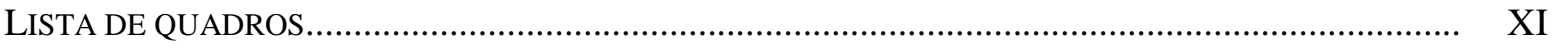

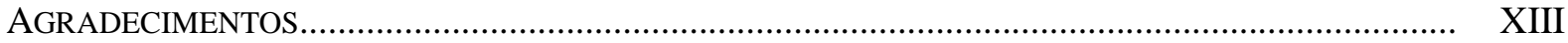

RESUMO

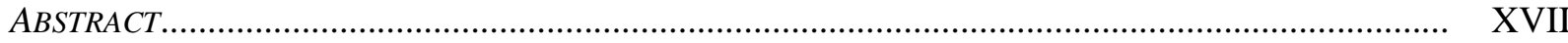

1. INTRODUÇÃ

1.1. PALEOCLIMA E PALEOGEOGRAFIA DA ANTÁRTICA DURANTE O CENOZOICO......................... 3

1.2. REGISTRO GEOLÓGICO DO CENOZOICO DA ANTÁRTICA OCIDENTAL................................... 9

1.3. FORAMINÍFEROS FÓSSEIS DO CENOZOICO DA ANTÁRTICA OCIDENTAL.................................. 11

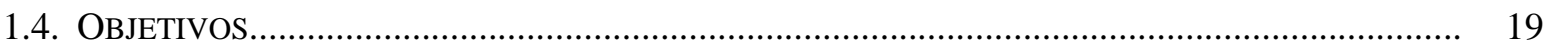

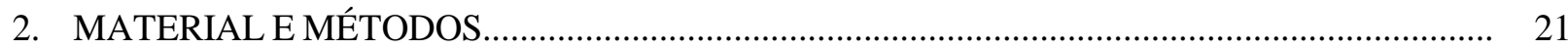

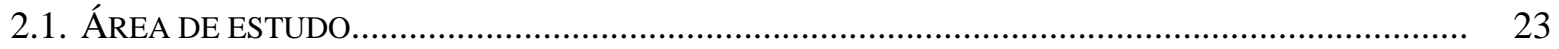

2.2. PROCEDIMENTOS EM LABORATÓRIO................................................................................... 29

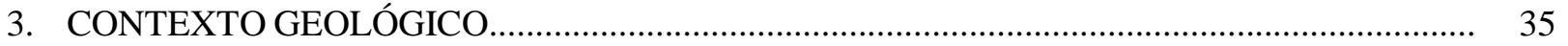

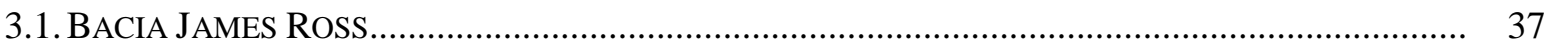

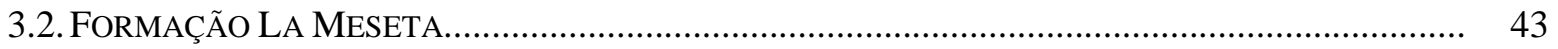

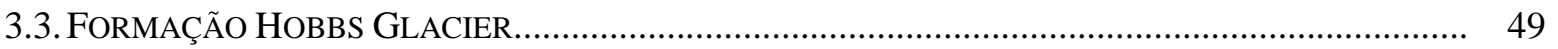

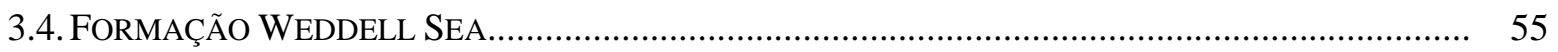

3.5. ESTRATIGRAFIA DA PORÇÃO NORDESTE DA ILHA SEYMOUR................................................... 59

4. BIOLOGIA E PALEOBIOLOGIA DE FORAMINÍFEROS................................................... 85

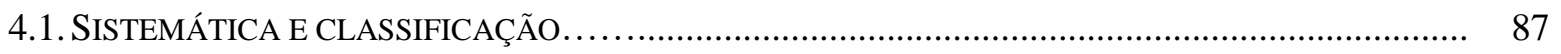

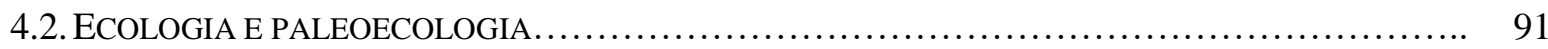

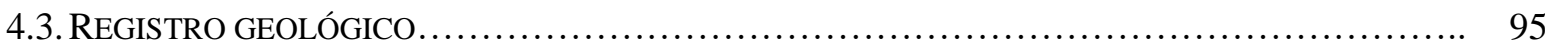

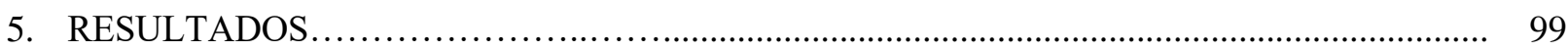

5.1. PETROGRAFIA DOS CALCÁRIOS DA FORMAÇÃo LA MESETA ….............................................. 101

5.2. DESCRIÇÕES DAS ASSEMBLEIAS DE MICROFÓSSEIS............................................................ 107

5.3. FEIÇÕES TAFONÔMICAS E PALEOECOLÓGICAS DAS ASSEMBLEIAS.......................................... 121

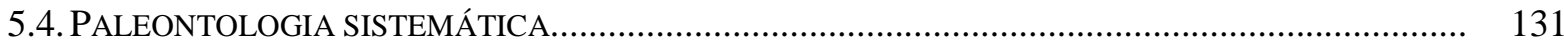

5.5. FORAMINÍFEROS E METAZOÁRIOS INDETERMINADOS...................................................... 225

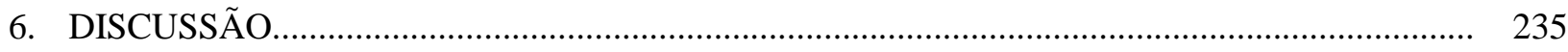

6.1. A TAFONOMIA DAS ASSEMBLEIAS E SEUS SIGNIFICADOS PALEOECOLÓGICOS........................ 237 
6.2. CORRELAÇÕES TAXONÔMICAS E SEUS SIGNIFICADOS PALEOBIOGEOGRÁFICOS....................... 263

6.3. FÓSSEIS-ÍNDICE E SUAS IMPLICAÇÕES PARA AS IDADES DOS DEPÓSITOS................................ 271

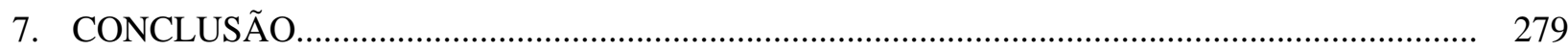

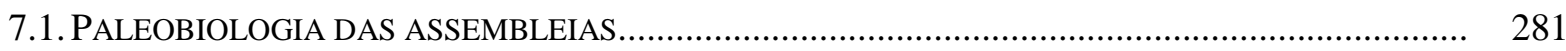

7.2. PaleOAMBIENTES DAS FormaÇÕES HobBS GLACIER E WEDDELL SEA................................. 283

7.3. IDADE DAS FORMAÇÕES HOBBS GLACIER E WEDDELL SEA.................................................. 285

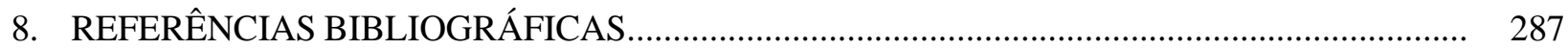
ANEXO I. LISTA DE LÂMINAS 


\section{RESUMO}

Os depósitos cenozoicos da Antártica Ocidental, especialmente aqueles da transição Eoceno-Oligoceno, fornecem importantes dados geológicos sobre as mudanças climáticas ocorridas ao longo da Era Cenozoica e de seu impacto na biosfera austral. Assembleias fósseis, incluindo de foraminíferos, foram relatadas para unidades de todas as épocas cenozoicas, em afloramentos dos arquipélagos James Ross e Shetlands do Sul. Todavia, os diamictitos das Formações Hobbs Glacier (Mioceno) e Weddell Sea (Plio-Pleistoceno), que afloram nas ilhas James Ross e Seymour, ainda não haviam sido alvo de análises micropaleontólogicas visando a obtenção de microfósseis com paredes inorgânicas. Foram analisadas amostras de 12 seções estratigráficas da Ilha Seymour, incluindo estratos do topo da Formação La Meseta (Eoceno) e de diversos níveis das formações Hobbs Glacier e Weddell Sea. Pela primeira vez foram encontradas assembleias de microfósseis com paredes inorgânicas, constituídas principalmente por foraminíferos, na porção superior da Formação La Meseta e em estratos das formações Hobbs Glacier e Weddell Sea. Na Formação La Meseta foram encontrados restos autóctones ou parautóctones de foraminíferos Textularia sp., primeira ocorrência do gênero para a unidade. Na Formação Hobbs Glacier, a assembleia autóctone ou parautóctone melhor preservada é composta pelo foraminífero lagenído Oolina stellula e pelo radiolário Larcopyle polyacantha. O foraminífero rotalíído Bolivina sp. é raro e representa um resto alóctone na unidade. Para a Formação Weddell Sea, a assembleia autóctone ou parautóctone melhor preservada é constituída pelo foraminífero lagenído Favulina hexagona e pelo planctônico Globigerinita uvula, além do rotaliído Globocassidulina subglobosa e do radiolário L. polyacantha nos mesmos e em outros níveis. Nos depósitos miocênicos e plio-pleistocênicos ocorrem também foraminíferos aglutinados grandes de táxons típicos de mar profundo, cujas feições tafonômicas indicam sua reelaboração a partir de depósitos mais antigos, possivelmente do Paleoceno, tendo em vista sua associação tafonômica e estratigráfica com o foraminífero Reticulophragmium garcilassoi, um fóssil-índice dessa época. Além de $R$. garcilassoi, ocorrem outros táxons típicos de assembleias de mar profundo na Formação Hobbs Glacier, como Alveolophragmium orbiculatum, Ammodiscus sp. nov., Ammodiscus pennyi, Ammomarginulina cf. aubertae, Bathysiphon sp. 1, Bathysiphon sp. 2, Cyclammina placenta e Nothia robusta. Na Formação Weddell Sea, as grandes formas aglutinadas são representadas por Ammodiscus sp. nov., Bathysiphon sp. 1, Budashevaella cf. laevigata, Cyclammina cancellata, Glomospira charoides, Saccammina grzybowski, Sculptobaculites barri e Verneulinoides cf. neocomiensis. Alguns táxons da Ilha Seymour podem ser associados àqueles dos depósitos paleocênicos da Nova Zelândia e Nova Guiné, sugerindo alguma correlação cronológica. Embora o registro fossilífero das formações La Meseta, Hobbs Glacier e Weddell Sea seja rarefeito, foi possível identificar restos autóctones ou parautóctones que indicaram a composição parcial das comunidades infaunais e planctônicas que habitavam a região durante a deposição das unidades. Os poucos fósseis-índice encontrados corroboram as idades já propostas paras as formações. Para a Formação Weddell Sea, as assembleias autóctones ou parautóctones e as formas planctônicas permitiram redefinir o contexto deposicional da unidade como glacio-marino, e não plenamente glacial, como anteriormente proposto.

Palavras-chave: microfósseis; foraminíferos; Eoceno; Mioceno; Plio-Pleistoceno; Antártica. 



\begin{abstract}
Western Antarctic deposits, especially those from the Eocene-Oligocene transition, provide important geological data on Cenozoic global climate changes and their impact on the southern biota. Fossil assemblages, including foraminifers, are known from geological units from all Cenozoic epochs, in outcrops of the James Ross and South Shetlands archipelagos. However, the diamictites of Hobbs Glacier (Miocene) and Weddell Sea (Plio-Pleistocene) formations, exposed in James Ross and Seymour islands, were never subjects of micropaleontologic analysis targeting inorganic-walled microfossils. Twelve stratigraphic sections on Seymour Island were analyzed, including the top of the La Meseta Formation (Eocene) and several strata of Hobbs Glacier and Weddell Sea formations. Assemblages of inorganic-walled microfossils, composed mainly of foraminifers, were found for the first time in the La Meseta Formation and in strata from the Hobbs Glacier and Weddell Sea formations. Autochthonous or parautochthonous remains of the foraminifer Textularia sp. were found in the La Meseta Formation, being the first occurrence of the genus in this unit. The best preserved autochthonous or parautochthonous assemblage from Hobbs Glacier Formation is composed of the Lagenid foraminifer Oolina stellula and radiolarian Larcopyle polyacantha. The Rotaliid foraminifer Bolivina sp. is rare and represents an allochthonous elements in this formation. In the Weddell Sea Formation, the best preserved autochthonous or parautochthonous assemblage is composed of the Lagenid foraminifer Favulina hexagona and the planktonic Globigerinita uvula, as well as the Rotaliid foraminifer Globocassidulina subglobosa and the radiolarian L. polyacantha in the same and in other strata. In these Miocene and Plio-Pleistocene deposits also occur large agglutinated foraminifers typical of the deep sea, whose taphonomic features indicate their reelaboration from older deposits, possibly from the Paleocene, given their taphonomic and stratigraphic association with the foraminifer Reticulophragmium garcilassoi, a Paleocene index-fossil. Besides $R$. garcilassoi, other typical deep-sea taxa occur in the Hobbs Glacier Formation, such as Alveolophragmium orbiculatum, Ammodiscus sp. nov., Ammodiscus pennyi, Ammomarginulina cf. aubertae, Bathysiphon sp. 1, Bathysiphon sp. 2, Cyclammina placenta and Nothia robusta. In the Weddell Sea Formation the agglutinated specimens are represented by Ammodiscus sp. nov., Bathysiphon sp. 1, Budashevaella cf. laevigata, Cyclammina cancellata, Glomospira charoides, Saccammina grzybowski, Sculptobaculites barri and Verneulinoides cf. neocomiensis. Some taxa from Seymour Island also occur in the Paleocene deposits of New Zealand and New Guinea, suggesting some chronological correlation. Although the fossil record of the La Meseta, Hobbs Glacier and Weddell Sea formations is sparse, it was possible to identify autochthonous or parautochthonous remains that indicate the partial composition of the infaunal communities and plankton that thrived in the area during the deposition of the units. The few index-fossils found corroborate the ages already indicated for the deposits. For the Weddell Sea Formation, the autochthonous or parautochthonous assemblages and the planktonic specimens allowed the redefinition of its depositional setting as glacial-marine, and not fully glacial, as previously proposed.
\end{abstract}

Keywords: microfossils; foraminifers; Eocene; Miocene; Plio-Pleistocene; Antarctica. 


\section{INTRODUÇÃO}





\subsection{Paleoclima e paleogeografia da Antártica durante o Cenozoico}

O último e atual clima glacial do planeta se estabeleceu durante a transição EocenoOligoceno, quando a tendência ao resfriamento global culminou na formação dos primeiros mantos de gelo permanentes sobre o continente antártico, principalmente em sua porção oriental. No entanto, a história paleoclimática da Era Cenozoica e da glaciação da Antártica é bastante complexa (Figura 1).

O planeta passou por dois períodos principais de aquecimento global anteriores à última glaciação antártica. O primeiro consiste no Máximo Termal do Paleoceno-Eoceno (MTPE), quando a temperatura média da superfície dos oceanos aumentou entre 5 e $7^{\circ} \mathrm{C}$, gerando mudanças nos padrões de circulação oceânica e anoxia de fundos oceânicos (Pearson, 2009; Bijl et al., 2010). Os foraminíferos bentônicos foram um dos principais grupos atingidos, com queda de diversidade maior do que aquela causada pela extinção Cretáceo-Paleogeno (Thomas, 1989; 1998). As causas do MTPE não são claras, mas as hipóteses incluem eventos capazes de elevar a concentração de $\mathrm{CO}_{2}$ na atmosfera, como aumento na atividade vulcânica, queima de turfeiras e desestabilização de hidratos de gás dos fundos oceânicos e subsequente liberação de gases estufa para a atmosfera, além de mudanças na excentricidade da órbita da Terra (cf. Prothero, 2009).

O Ótimo Termal do Eoceno Médio (OTEM) representa o segundo evento de intenso e rápido aquecimento, quando a temperatura média das águas profundas se elevou entre 4 e $5{ }^{\circ} \mathrm{C} \mathrm{e}$ os níveis de $\mathrm{CO}_{2}$ atmosférico chegaram a $4600 \mathrm{ppm}$ (partes por milhão), seu pico durante o Cenozoico (Bohaty \& Zachos, 2005; Zachos et al., 2008; Galazzo et al., 2014). As causas do aumento dos níveis de $\mathrm{CO}_{2}$ e consequente aquecimento são desconhecidas, mas uma das hipóteses é que uma reativação do vulcanismo responsável pela Grande Província Ígnea do Deccan, na Índia, gerou fluídos hidrotermais que dissolveram calcários depositados anteriormente em plataformas carbonáticas do Mar de Tétis e, consequentemente, liberaram enormes quantidades de $\mathrm{CO}_{2}$ para atmosfera (Pearson, 2010).

O estabelecimento do clima glacial, com vasta cobertura de gelo nos polos, ocorreu logo após e não se deu apenas pela posição geográfica do continente, mas por outros fatores que, em sinergia, contribuíram para o resfriamento não apenas da Antártica, mas do clima global.

Inicialmente, a hipótese era de que apenas a separação da Antártica, América do Sul, Austrália e Nova Guiné, há aproximadamente $40 \mathrm{Ma}$, teria causado o isolamento térmico do 
continente devido à formação da Corrente Circumpolar Antártica, que impediria que correntes oceânicas aquecidas nas áreas intertropicais distribuíssem parte de seu calor na região polar (e.g., Toggweiler \& Bjornsson, 2000; Scher \& Martin, 2006) (Figura 2). De fato, as aberturas da Passagem de Drake e do Mar da Tasmânia e a formação da Corrente Circumpolar Antártica, entre o Eoceno Inicial e Médio, coincidem com o início da tendência de resfriamento do clima global, como indicado pelos isótopos de oxigênio dos depósitos dos oceanos profundos; mas estes eventos antecedem a formação da calota polar antártica em pelo menos $5 \mathrm{Ma}$, indicando não serem eles os responsáveis pela glaciação antártica (Lagabrielle et al., 2009).

Diversos estudos publicados nos anos 2000 (e.g., DeConto \& Pollard, 2003; Pagani et al., 2005; Huber \& Nof, 2006; Pearson et al., 2009) demonstraram a sincronicidade entre o desenvolvimento da calota polar antártica e a redução da concentração de $\mathrm{CO}_{2}$ na atmosfera, sugerindo ser este o evento crítico.

Os níveis de $\mathrm{CO}_{2}$ atmosférico caíram rapidamente a partir da transição Eoceno-Oligoceno, quando a concentração passou de 1000 ppm para 400 ppm (Pagani et al., 2005), sendo que os primeiros mantos de gelo só se formaram quando a concentração de $\mathrm{CO}_{2}$ atingiu 760 ppm, no início do Oligoceno (Pearson et al., 2009). Isto marca, portanto, uma rápida transição de um planeta em condições de "efeito-estufa" (greenhouse) para um planeta em condições de "efeito-geladeira" (icehouse).

O final do OTEM coincide com constantes florações (blooms) de pteridófitas aquáticas do gênero Azolla nos oceanos Ártico e Nórdico, entre aproximadamente 48 e 49,4 Ma, na transição Eoceno Inicial-Eoceno Médio (Brinkhuis \& Schouten, 2006). Essas florações foram frequentes ao longo de aproximadamente um milhão de anos, como evidenciado por depósitos métricos onde restos autóctones dessas pteridófitas e matéria orgânica associada são extremamente abundantes, depositados durante um período em que a salinidade das águas superficiais era muito baixa (cf. Brinkhuis \& Schouten, 2006; Waddell \& Moore, 2008; Gleason et al., 2009; Speelman et al., 2009; Barke et al., 2012). Este processo, ocorrendo em toda a área dos oceanos Ártico e Nórdico e com a matéria orgânica das plantas sendo soterrada nos fundos oceânicos, seria capaz de sequestrar enormes quantidades de carbono, suficientes para reduzir a concentração de $\mathrm{CO}_{2}$ em até $80 \%$ (cf. Waddell \& Moore, 2008; Speelman et al., 2009). Entretanto, embora este possa ter sido o gatilho inicial do resfriamento global, também é provável que variações na órbita da Terra tenham 
favorecido a ocorrência de verões com temperaturas médias relativamente mais baixas, contribuindo para a instalação de um clima glacial (DeConto \& Pollard, 2003).

Embora a glaciação da Antártica tenha se iniciado na transição Eoceno-Oligoceno, como evidenciado por depósitos do Oligoceno Inicial da porção oriental do continente (Zachos et al, 1992; Sorlien et al., 2007), os mais antigos depósitos periglaciais ou próglaciais da Antártica Ocidental são datados do Oligoceno Médio ou do Oligoceno Tardio (Santos, 1996; Troedson \& Smelie, 2002). Isto, portanto, sugere que detalhes da história glacial da Antártica Ocidental foram diferentes daqueles da Antártica Oriental, onde geleiras teriam se formado apenas milhões de anos após o início da glaciação no continente.

Mesmo com a rápida queda de temperatura e consequente glaciação após a transição Eoceno-Oligoceno, a temperatura global continuou a sofrer variações, com períodos de aquecimento global marcando o Oligoceno Tardio e o Mioceno Médio (Zachos et al., 2001). O último, conhecido com Ótimo Climático do Mioceno Médio (OCMM), é caracterizado por uma temperatura global média de $18,4^{\circ} \mathrm{C}$ - aproximadamente $3{ }^{\circ} \mathrm{C}$ mais alta que a média atual -, com águas oceânicas profundas e águas superficiais de altas latitudes relativamente quentes (cf. Zachos et al., 2008; Shevenell et al., 2008; Pound et al., 2012). Todavia, as causas deste aquecimento ainda são desconhecidas. A concentração de $\mathrm{CO}_{2}$, de acordo com isótopos (e.g., You et al., 2009; Zhang et al., 2013) e com a contagem de estômatos de plantas terrestres (Kurshner et al., 2008), era de aproximadamente 500 ppm, taxa que, por si mesma, não causaria o aumento e a manutenção de uma temperatura média tão alta. Assim, é necessário inferir condições simultâneas de El Niño, para o aquecimento das águas pacíficas, e mudanças na obliquidade da Terra, para gerar verões mais quentes (Goldner et al., 2014).

Por fim, o planeta retomou sua tendência ao resfriamento entre o Mioceno Tardio e o Plioceno, tornando-se progressivamente mais frio, seco e marcadamente sazonal. Durante o Plioceno, no entanto, a temperatura superficial média era aproximadamente 2 a $3{ }^{\circ} \mathrm{C}$ mais alta que a atual (Robinson et al., 2008); e, ao final dessa época, o soerguimento do Istmo do Panamá interrompeu a circulação oceânica entre os oceanos Atlântico e Pacífico. Isto causou o aquecimento momentâneo do Atlântico e, consequentemente, o aumento da umidade atmosférica, a qual serviu de matéria prima para o crescimento dos recém-formados mantos de gelo da Groelândia, culminando no último período glacial da Era Cenozoica (Bartoli et al., 2005; Haywood et al., 2009). 

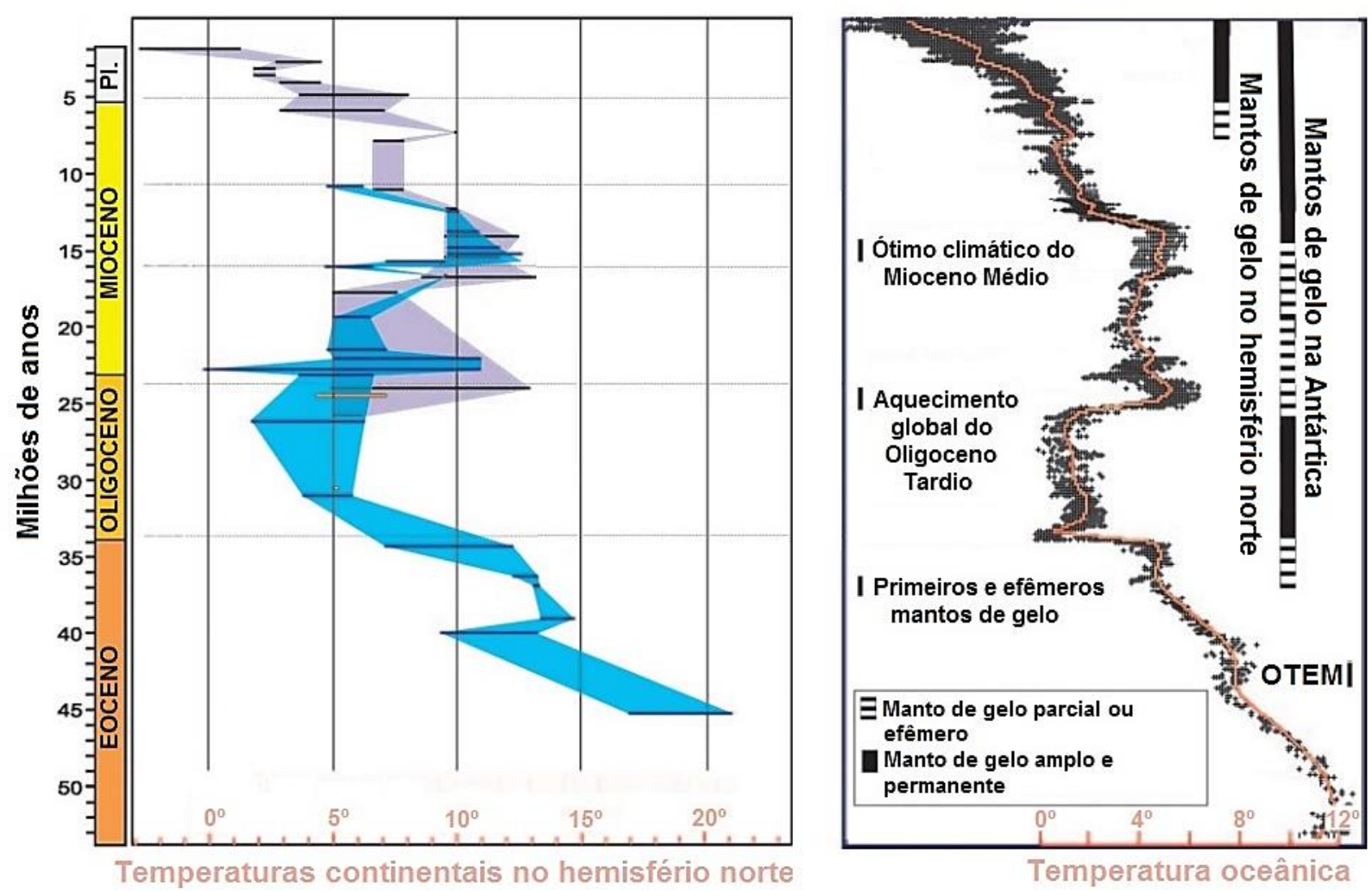

Figura 1. Variações nas temperaturas médias de continentes e oceanos e mantos de gelo no hemisfério norte e na Antártica durante o final do Paleogeno e início do Neogeno. OTEM: Ótimo Climático do Eoceno Médio. (Modificado de Mosbrugger et al. [2005].) 


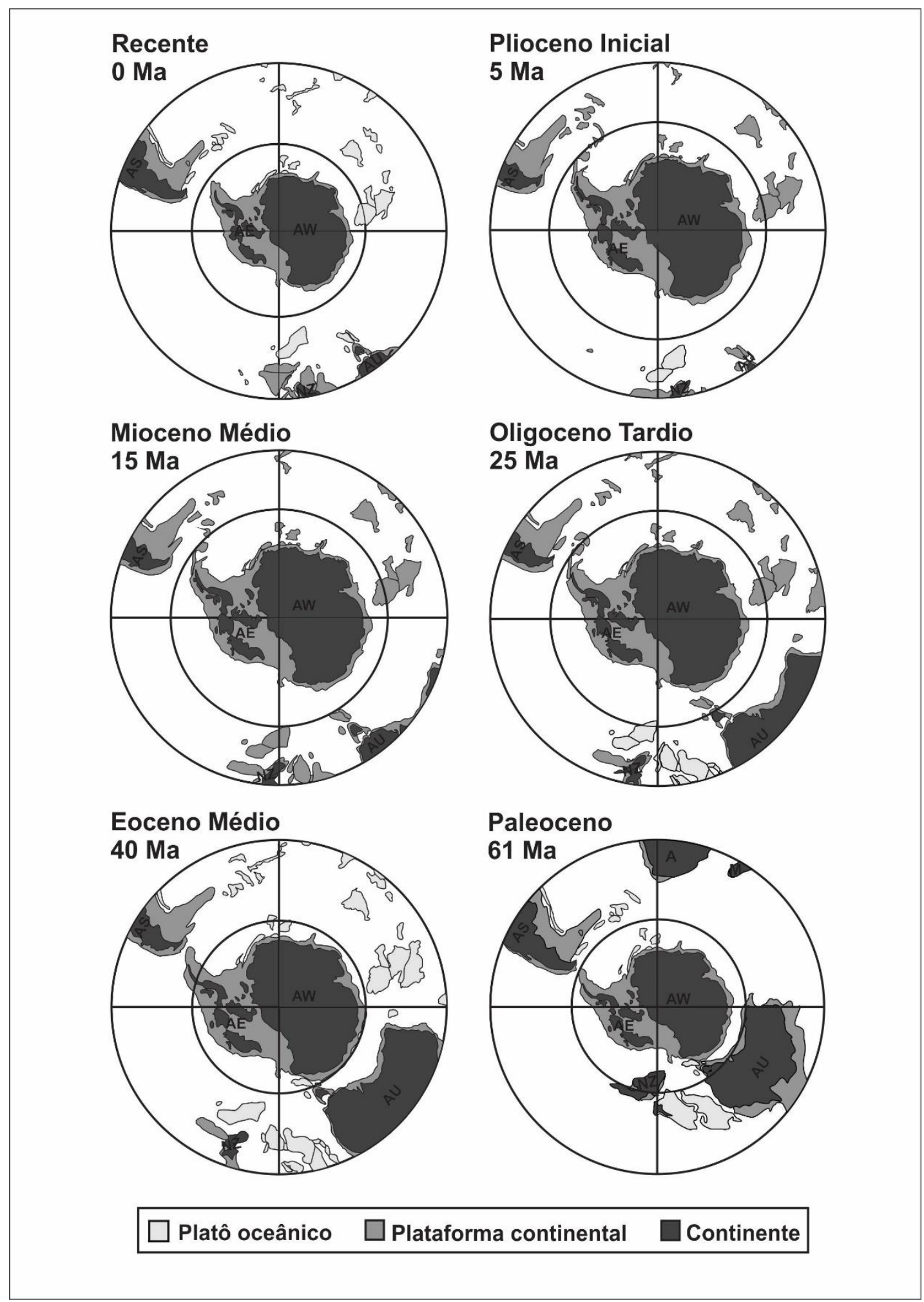

Figura 2. Paleogeografia da região polar sul durante a Era Cenozoica. Mantos de gelo foram omitidos. (Redesenhado a partir de Lawver \& Gahagan [2003] e Brown et al. [2006].) 



\subsection{Registro geOlógico do CenOzoico da AnTÁrtica OCIDENTAL}

A maior parte da história geológica das mudanças climáticas da Antártica durante a Era Cenozoica é conhecida através de testemunhos de depósitos marinhos, os quais registraram as condições de ambientes oceânicos relativamente profundos (e.g., Barron \& Larsen, 1989; Breza \& Wise, 1992; Francis et al., 2009; Barrett, 2009). O registro de ambientes costeiros, por sua vez, se encontra acessível nos afloramentos de rochas sedimentares do continente e, principalmente, de suas ilhas.

Embora a Antártica possua uma vasta área, poucas unidades geológicas cenozoicas são conhecidas, uma vez que os mantos de gelo recobrem quase totalmente a superfície do continente. As principais unidades descritas se localizam na porção norte da Península Antártica - com a importante exceção dos depósitos neopaleozoicos e triássicos das Montanhas Transantárticas (Hamilton \& Hayes, 1963; Craddock, 1982) -, onde o clima relativamente mais quente, estabelecido após o início do atual período interglacial, expôs rochas ígneas e sedimentares. As áreas onde afloramentos foram descritos incluem algumas ilhas do Arquipélago das Shetlands do Sul, especialmente Ilha King George, e o Arquipélago James Ross - denominação coletiva e informal para designar a Ilha James Ross e suas ilhas vizinhas, Cockburn, Lockyer, Seymour, Snowhill e Vega - e a parte adjacente da Península Trinity (cf. Birkenmajer, 1980; Davies, 2014).

No Arquipélago das Shetlands do Sul, as principais unidades sedimentares cenozoicas foram descritas para a Ilha King George, sendo elas as formações Destruction Bay (Oligoceno) e Cape Melville (Mioceno), do Grupo Moby Dick, e a Formação Polonez Cove (Oligoceno) (cf. Birkenmajer, 1980; Birkenmajer et al., 1983; Dingle et al., 1997; Troedson \& Smellie, 2002). Cabe ressaltar que, até o momento, não existe proposta consensual de divisão litoestratigráfica para a Ilha King George (Cantrill \& Poole, 2012).

Os principais depósitos sedimentares cenozoicos do Arquipélago James Ross incluem as formações López de Bertodano (Cretáceo Tardio-Paleoceno), Sobral (Paleoceno) e Cross Valley (Paleoceno), do Grupo Marambio; a Formação La Meseta (Eoceno), do Grupo Ilha Seymour; a Formação Hobbs Glacier (Mioceno Tardio), do Grupo Vulcânico Ilha James Ross; a Formação Ilha Cockburn (Plioceno); e a Formação Weddell Sea (Plio-Pleistoceno) (cf. Sadler, 1988; Pirrie et al., 1992; Pirrie et al., 1997a; Pirrie et al., 1997b; Gazdzicki et al., 2004; Stilwell, 2002; Marenssi et al., 2010; Marenssi et al., 2012). 
Ivany et al. (2006) relataram um pretenso diamictito datado da transição Eoceno-Oligoceno ou do Oligoceno Inicial na Ilha Seymour, sotoposto pela Formação La Meseta e sobreposto pela Formação Weddell Sea, Todavia, a ocorrência do diamictito, na litologia e contexto estratigráfico descritos, e a suposta idade oligocênica não foram, respectivamente, confirmadas e corroboradas por estudos posteriores (e.g., Concheyro et al., 2007; Marenssi et al., 2009).

Fósseis foram relatados para todas as unidades cenozoicas citadas anteriormente. Para a Ilha Rei George, foram descritas assembleias fósseis abundantes e diversas, compostas principalmente por moluscos bivalves, nas formações Polonez Cove e Cape Melville (cf. revisão de Quaglio, 2007), além de assembleias de foraminíferos bentônicos e planctônicos (Birkenmajer \& Luczkowska, 1987; Gazdzicki, 1989; Majewski \& Gazdzicki, 2014). Para o Arquipélago James Ross, por sua vez, também foram descritas abundantes e diversas assembleias de invertebrados, vertebrados e plantas nos depósitos da Formação La Meseta, que aflora nas ilhas Seymour e Cockburn e representa a unidade mais fossilífera conhecida no continente (Stilwell \& Zinsmeister, 1992). Foraminíferos bentônicos e planctônicos também foram descritos para a unidade, mas foram encontrados apenas em sua base (Gazdzicki \& Majewski, 2012).

Os depósitos periglaciais ou próglaciais das formações Hobbs Glacier e Weddell Sea, também na área do Arquipélago James Ross, contêm raros restos de invertebrados e abundantes palinomorfos, estes últimos geralmente representando espécimes retrabalhados dos depósitos sotopostos (Pirrie et al., 1997b; Gazdzicki et al., 2004). De fato, as duas unidades nunca foram alvo de análises micropaleontológicas que visassem a obtenção de microfósseis de paredes inorgânicas - ou, se foram, os resultados de tais análises jamais foram publicados.

Por fim, a Formação Ilha Cockburn contém os últimos registros de invertebrados dos depósitos cenozoicos da Antártica Ocidental, representados por invertebrados relativamente abundantes e diversos (cf. revisão de Gazdzicki, 1993), além de foraminíferos e alguns microfósseis de paredes inorgânicas (Gazdzicki \& Webb, 1995). 


\subsection{Foraminíferos fósSeis do Cenozoico da AnTártica OCIDENTAL}

Relativamente poucas assembleias de foraminíferos foram descritas para os depósitos cenozoicos da Antártica Ocidental, tendo sido relatadas em 11 estudos (i.e., Huber, 1986; Birkenmajer \& Luczkowska, 1987; Huber, 1988; Gazdzicki, 1989; Gazdzicki \& Webb, 1996; Strong \& Webb, 2000; Gazdzicki \& Majewski, 2012; Majewski et al., 2012; Caramés \& Concheyro, 2013; Concheyro et al., 2014; Majewski \& Gazdzicki, 2014). A maioria dessas ocorrências foi descrita para afloramentos na Península Antártica, sobretudo para algumas ilhas do Arquipélago James Ross e para a Ilha King George do Arquipélago das Shetlands do Sul; e, embora o registro seja rarefeito, ele se distribui por todas as épocas do Cenozoico, com exceção do Holoceno.

\section{Assembleias do Paleoceno: Formações lópez de Bertodano e Sobral, ARQuiPÉlago James Ross}

Huber (1986) foi o primeiro a descrever foraminíferos fósseis do Arquipélago James Ross, relatando formas planctônicas para a transição Cretáceo-Paleogeno, registrada na Formação López de Bertodano, e, poucos metros acima, para a Formação Sobral, do Paleoceno, nas ilhas James Ross, Seymour e Vega. Porém, as assembleias de ambas as unidades foram descritas, em sua totalidade e em detalhe, apenas por Huber (1988).

As assembleias de Huber (1988) são caracteristicamente bentônicas e se separam por uma fácies de dissolução típica do Paleoceno Inicial de médias e baixas latitudes austrais, já relatada para a Nova Zelândia (Webb, 1971; Strong, 1984), extremo sul da América do Sul (Krashennikov \& Basov, 1986) e sul do Atlântico Sul (Hsü, 1984).

As assembleias da Formação López de Bertodano são compostas principalmente por foraminíferos bentônicos calcários, sendo dominadas por Buliminella procera Huber, 1988, Chilostomella subtriangularis (Kline, 1943) (relatado como Allomorphina subtriangularis Kline, 1984 pelo autor) e Nonionella sp. As raras formas aglutinadas são representadas por Hyperammina elongata Brady, 1878 e Reophax sp., e as raríssimas formas planctônicas foram identificadas como 
Globoconusa daubjergensis (Brönnimann, 1953) (relatada como Globastica daubjergensis Brönnimann 1952 pelo autor), um fóssil-índice para o Daniano.

As assembleias da Formação Sobral também são caracteristicamente bentônicas, mas são dominadas por B. procera, que representa $82 \%$ dos espécimes. A espécie planctônica $G$. daubjergensis também está presente, mas é pouco comum. Estas assembleias se assemelham à anterior pela predominância de B. procera e pela ocorrência de G. daubjergensis e de Nonionella sp., além de mais três espécies raras, e se diferenciam pela presença de formas aglutinadas na primeira, sua ausência na segunda e por 18 táxons que são exclusivos de uma ou de outra assembleia.

Cabe ressaltar ainda que foraminíferos aglutinados, ostracodes, micromoluscos, espinhos de equinoides e calcisferas, quando ocorrem, são pouco comuns ou raros e mal preservados nos depósitos danianos da porção superior da Formação López de Bertodano e da Formação Sobral, o que contrasta com os depósitos inferiores, de idade cretácica, da Formação López de Bertodano, nos quais foraminíferos aglutinados são muito comuns e diversos (Huber, 1988).

\section{AsSembleias do Eoceno: FormaÇão la Meseta, IlHa Seymour}

Gazdzicki \& Majewski (2012) descreveram as primeiras e, até o momento, únicas assembleias eocênicas conhecidas para a Antártica Ocidental, provenientes da base da Formação La Meseta na Ilha Seymour, todas caracteristicamente bentônicas e calcárias. (Contudo, cabe mencionar que Bitner [1996] relatou a ocorrência de raros espécimes de Cibicides sp. incrustados em conchas de braquiópodes na mesma unidade.)

A primeira assembleia (ZPAL2) é dominada por ?Nonionellina sp., mas também são comuns espécimes de Eilohedra vitrea (Parker, 1953), Globocassidulina subglobosa (Brady, 1881), Nonionoides turgida (Williamson, 1858) (relatada pelos autores como Nonionella bradyi Williamson, 1858) e Cibicides williamsoni Garrett, 1941. A segunda assembleia (ZPAL5), por sua vez, é dominada por G. subglobosa e Cribroelphidium aff. lauritaense (Todd \& Kniker, 1952), mas também são comuns espécimes de Guttulina communis (d'Orbigny, 1826) (relatada pelos autores como Guttulina irregularis [d'Orbigny, 1826]) e Lobatula sp. A terceira e última assembleia (ZPAL11) é composta apenas por três espécimes de foraminíferos planctônicos 
indeterminados, um espécime de Cibroelphidium sp. e outro espécime de Cibroelphidium aff. saginatum (Finlay, 1939).

As assembleias ZPAL2 e ZPAL5, portanto, são dominadas por formas hialinas bentônicas, com táxons característicos de ambientes de plataforma continental interna, laguna e estuário de clima temperado. Além disso, a ausência de espécies planctônicas em ZPAL2 e ZPAL5 e sua raridade em ZPAL11 indicam que o corpo d'água no qual habitavam era consideravelmente restrito. No mesmo sentido, a maior proporção de Cibroelphidium aff. saginatum em ZPAL5 (em torno de $30 \%$ ), quando comparada com sua proporção nas associações modernas da Nova Zelândia (em torno de 10\%), também sugerem um corpo d'água ao menos parcial ou sazonalmente restrito.

\section{Assembleias do Oligoceno: Formação Polonez Cove, Ilha King George}

Os primeiros foraminíferos oligocênicos da Antártica Ocidental foram descritos por Gazdzicki (1989), que os encontrou em coquinas de bivalves pectenídeos do Membro Low Head da Formação Polonez Cove (sensu Birkenmajer, 1980) - equivalente ao "Conglomerado Pecten" de Barton (1965) e ao "Conglomerado suportado por Chlamys" de Gazdzicki (1989) -, na Ilha King George. A assembleia é particularmente interessante porque, durante o período em que foi depositada, mantos de gelo continental se estendiam da Península Antártica até a Ilha King George (Birkenmajer, 2001; Troedson \& Smellie, 2002).

As assembleias de Gazdzicki (1989) são extremamente pobres, com 13 espécimes para 10 kg de rocha, e todos seus táxons são planctônicos, sendo eles Subbotina angiporoides (Hornibrook, 1965) (relatada como Globigerina angiporoides Hornibrook, 1965), Chiloguembelina cubensis (Palmer, 1934) e Globorotalia sp. Os espécimes de $S$. angiporoides e C. cubensis podem ser correlacionadas às formas planctônicas do Oligoceno Inicial da Australia, Nova Zelândia e sul dos oceanos Atlântico e Pacífico (Gazdzicki, 1989). (Cabe ressaltar que Gazdzicki [1989] citou ter encontrado assembleias bentônicas diversas e abundantes, com membros das famílias Cassidulinidae, Cibicidae, Elphidiidae, Glandulinidae, Miliolidae e Nodosariidae, cujos espécimes estavam sendo preparados pelo autor. Porém, novos foraminíferos da Formação Polonez Cove seriam descritos mais de duas décadas depois, por Majewski \& Gazdzicki [2014].) 
Majewski \& Gazdzicki (2014) relataram assembleias densas e diversas que parecem representar uma única comunidade de foraminíferos, a maioria representantes das ordens Lagenida, Miliolida e Rotaliida, com raras formas aglutinadas das ordens Lituolida. Spirillinida e Textulariida.

As assembleias de Majewski \& Gazdzicki (2014) são dominadas por Cibicides temperata Vela, 1957, que compõe mais da metade do número total de espécimes encontrados. Outros táxons importantes são, em ordem decrescente de abundância, Globocassidulina subglobosa (Brady, 1881), Discorbis sp., Cibicidoides lobatulus (Walker \& Jacob, 1878) (relatada pelos autores como Lobatula lobatula [Walker \& Jacob, 1798]), Ammoelphidiella sp., Melonis sp. e Triloculina spp. As raríssimas formas aglutinadas correspondem a ocorrências singulares de Ammodiscus planorbis Höglund, 1947, Siphotextularia finlayi Hornibrook, 1961 e ?Trochammina sp.

Majewski \& Gazdzicki (2014) atribuem a escassez de formas aglutinadas a um viés tafonômico, o qual seria indicado pela predominância de formas calcárias com testas robustas, mais resistentes à dissolução. Os autores ressaltam ainda que, conforme relatado por estudos como aquele de Schröder (1988), formas aglutinadas de depósitos recentes são comumente desagregadas já no início da diagênese, e que o tratamento de desagregação com sulfato de sódio decahidratado (ou sal de Glauber) é quimicamente agressivo e pode desagregar boa parte das formas aglutinadas de uma amostra.

Por fim, embora as assembleias ocorram em fácies de frente de leque deltaico, diversos táxons são característicos de ambientes neríticos relativamente rasos da Antártica Ocidental. Mas, de modo geral, as assembleias não são correlacionáveis a outras assembleias fósseis ou atuais da Antártica, talvez por representarem uma comunidade de ambientes rasos que não fora preservada em outras unidades do continente, uma vez que a maioria das assembleias provém de contextos marinhos profundos (Majewski \& Gazdzicki, 2014).

\section{Assembleias do Mioceno: Formação Cape Melville, IlHa King George, E DEPÓSITOS DO MAR DE WEDDELL}

Assembleias de foraminíferos tipicamente miocênicas foram relatadas por Birkenmajer \& Luczkowska (1987) para a Formação Cape Melville, do Mioceno Inicial da Ilha King George, e 
por Majewski et al. (2012) para depósitos marinhos do Mioceno Médio do extremo norte do Mar de Weddell.

Embora os foraminíferos relatados por Birkenmajer \& Luczkowska (1987) sejam, de modo geral, mal preservados (ao ponto de aproximadamente $50 \%$ dos espécimes serem indeterminados), os autores foram capazes de definir duas "assembleias-zona" ao analisarem 24 assembleias, com distribuição estratigráfica bem definida: (1) assembleia-zona A, ou assembleia CibicidesCibicidoides, característica da porção inferior da formação, na qual os táxons dominantes são Cibicides refulgens Montfort, 1808 e Cibicidoides aff. pseudoungerianus (Cushman, 1922), com importante contribuição de outras espécies dos mesmos gêneros; e (2) assembleia B, ou assembleia Cribrostomoides-Cyclammina-Globobulimina, característica da porção superior da formação, na qual os táxons dominantes são Cribrostomoides crassimargo (Norman, 1892), Globobulimina auriculata (Bailey, 1894) e diversas espécimes de Cyclammina. Cabe citar que não foram encontrados foraminíferos planctônicos e que, em ambas as assembleias, são comuns radiolários esféricos e fragmentos de colônias de briozoários e de equinodermos.

Na base da formação, as assembleias Cibicides-Cibicidoides apresentam táxons típicos de ambientes profundos, com predominância de formas aglutinadas, incluindo Ammodiscus sp. e Rhabdammina sp. Em direção ao topo, na porção média da unidade, tanto a assembleia A quanto a assembleia B passam a ter composição mista ou predominantemente calcária, com composição e proporção taxonômica típica de ambientes de plataforma rasa. Todavia, as assembleias voltam a se tornar predominantemente aglutinadas no topo da formação, indicando mais uma tendência à elevação do nível de base e paleoambientes batiais (Birkenmajer \& Luczkowska, 1987).

A litologia dos folhelhos, clastos caídos e fósseis de invertebrados e de foraminíferos indicam deposição em ambientes relativamente profundos, de plataforma rasa a batiais, sempre abaixo do nível de onda e sofrendo influência de blocos de gelos que transportavam e liberavam clastos e grandes blocos erráticos para o fundo (cf. Birkenmajer, 1982a; 1984; 1989).

A assembleia relatada por Majewski et al. (2012) é consideravelmente menos densa que aquela relatada acima. Foram identificados sete táxons na assembleia mais densa e diversa, sendo o mais comum Elphidium cf. macellum (Fichtel \& Moll, 1798), seguido por ?Triloculina sp., Loeblichopsis sabulosa (Brady, 1882) e ?Biasterigerina sp. Formas aglutinadas são extremamente raras e a maioria ocorre como fragmentos, sendo reconhecível apenas L. sabulosa. Também ocorrem associados microgastrópodes, veliconchas de bivalves e ostracodes. 
Majewski et al. (2012) comentam que, embora provavelmente retrabalhados, os foraminíferos e fósseis associados possuem a mesma idade da rocha matriz, tendo em vista a ocorrência de E. macellum, um táxon tipicamente miocênico. Segundo os autores, a predominância de E. aff. macellum e de miliolídeos e a ausência de foraminíferos planctônicos (e de outros organismos planctônicos em geral) sugerem um paleoambiente raso, possivelmente na zona de inframaré ou de entremaré. Os autores argumentam ainda que a ambiguidade taxonômica (i.e., a singularidade da composição taxonômica) de algumas assembleias pode representar não um artefato de informação - tendo em vista os poucos registros sobre assembleias fósseis de foraminíferos da Antártica Ocidental -, mas a proximidade entre diferentes comunidades de diferentes ambientes que existiam próximos ao continente durante o Mioceno Médio.

\section{Assembleias do Plioceno: Formação Ilha Cockburn, IlHa CoCKburn}

Foraminíferos foram descritos para a Formação Ilha Cockburn por Holland (1910), Hennig (1910), Gazdzicka \& Gazdzicki (1994) e Gazdzicki \& Webb (1995). Todavia, Holland (1910) e Henning (1910) relataram poucos espécimes, provenientes de uma única amostra e com identificações taxonômicas pouco consistentes, enquanto Gazdzicki \& Gazdzicki (1994) relataram formas aglutinadas e planctônicas que foram exumadas dos depósitos cretácicos e redepositadas nos depósitos pliocênicos. Assim, será exposto aqui apenas o estudo de Gazdzicki \& Webb (1995), que, ao relatar novos táxons, também os comparou com aqueles de Holland (1910) e revisou sua taxonomia.

Gazdzicki \& Webb (1995) relataram assembleias dominadas por representantes das famílias Cassidulinidae, Cibicididae e Elphidiidae. As diversas assembleias parecem representar uma única associação (i.e., uma biocenose ou comunidade), tendo em vista que, em todas elas, 90\% dos espécimes correspondem à Globocassidulina crassa (d'Orbigny, 1839) (relatada pelos autores como Cassidulina crassa d'Orbigny, 1839), Globocassidulina subglobosa (Brady, 1881), Cassidulinoides parkerianus (Brady, 1881), Lobatula lobatula (Walker \& Jacob, 1798) (relatada pelos autores como Cibicides lobatulus [Walker \& Jacob, 1798]) e Ammoelphidiella antarctica (Conato \& Segre, 1974). Porém, apenas uma das assembleias parece representar com alguma fidelidade a biocenose original e in situ, tendo em vista a ocorrência de muitos espécimes bem 
preservados, diversidade mais alta e espécimes de um mesmo táxon com testas de diferentes gerações, diferentes estágios ontogenéticos e diferentes tamanhos. Além disso, também foram encontrados outros microfósseis associados, como diatomáceas, radiolários, escleritos de poríferos, ostracodes, briozoários e fragmentos de moluscos e de equinoides.

A presença de A. antarctica, um fóssil-índice para os depósitos pliocênicos costeiros das bacias antárticas, e, secundariamente, a predominância de membros da Família Cassidulinidae permitem correlacionar a Formação Ilha Cockburn com a Formação Prospect Mesa, do Vale Wright, e com os depósitos pliocênicos das Colinas Larsemann, ambos na Antártica Oriental (cf. Webb, 1974).

Por fim, as assembleias de foraminíferos, os invertebrados e a litologia das coquinas indicam um paleoambiente costeiro de alta energia, com profundidade máxima de 50 metros, sem qualquer evidência de mantos de gelo permanentes ou sazonais, indicando deposição durante períodos interglaciais ou de degelo.

\section{Assembleias do Pleistoceno: Diamictitos da IlHa Vega}

A Ilha Vega possui alguns dos depósitos mais recentes do Arquipélago James Ross, datados do Pleistoceno. Eles são representados por arenitos, diamictitos e brechas hialoclásticas, sugerindo a deposição em um delta de till ou em bancos de morenas subaquáticas e em áreas de escorregamento próximas à base de geleiras (Smellie et al., 2006; Hambrey \& Smellie, 2006). As assembleias de foraminíferos foram descritas para os diamictitos e brechas por Caramés \& Concheyro (2013) e Concheyro et al. (2014).

Caramés \& Concheyro (2013) relataram assembleias bentônicas, quase exclusivamente calcárias, para diversos níveis de diamictitos da localidade Cape Lamb, na extremidade sul da Ilha Vega. Os táxons mais comuns são os infaunais Globocassidulina biora (Crespin, 1960), Cribroelphidium aff. excavatum (Terquem, 1875) (relatado como Cibroelphidium sp. aff. E. excavatum pelos autores) e Cassidulinoides parkeriana (Brady, 1881) (relatado como Cassidulinoides parkerianus [Brady, 1881] pelos autores), mas também é comum o epifaunal Cibicides refulgens Montfort, 1808. Essas assembleias ocorrem associadas a pectinídeos e braquiópodes muito bem preservados. 
A composição taxonômica das assembleias é típica de ambientes de plataforma interna, o que se coaduna com a interpretação de um contexto deposicional de bancos de morenas subaquosas aventada por Smellie et al. (2006)

Concheyro et al. (2014), por sua vez, relataram assembleias extremamente pobres para alguns níveis dos diamictitos da região central da Ilha Vega, nas localidades de Leal Bluff e Sandwich Bluff.

Em Sandwich Bluff ocorrem duas assembleias, a primeira composta por um espécime de Cibicides sp. e outro de Gavellinella sp. e a segunda representada apenas por um espécime mal preservado de Pullenia jarvisi Cushman, 1936. Esta é a primeira ocorrência de Gavellinella sp. para a Antártica, mas o gênero é extremamente comum em depósitos cretácicos de quase todos os oceanos e continentes, tornando-se raro a partir do início da Era Cenozoica, o que sugere que o espécime foi sido exumado dos depósitos cretácicos da região e redepositados nos diamictitos.

A assembleia da localidade de Leal Bluff, é composta por um espécime de Dorothia sp., outro bem preservado de Trochammina aff. intermedia Rhumbler, 1938 (relatado pelos autores como Trochammina sp. aff G. intermosia) e por dezenas de palinoforaminíferos (i.e., revestimentos quitino-proteícos internos de foraminíferos) e seus fragmentos, alguns com preservação excepcional, mostrando os revestimentos internos de prolóculos.

Em ambas as localidades os foraminíferos ocorrem associados a nanofósseis calcários e cistos de dinoflagelados típicos de depósitos cretácicos, comparáveis aqueles das formações Ilha Snow Hill e López de Bertodano, que também afloram na ilha. Com exceção desses restos, a única correspondência entre os diamictitos pleistocênicos do centro da ilha com aquelas da extremidade sul é a presença de $C$. refulgens. 


\subsection{OBJETIVOS}

Com vistas a ampliar o conhecimento sobre os foraminíferos e microfósseis associados das formações La Meseta (Eoceno), Hobbs Glacier (Mioceno) e Weddell Sea (Plio-Pleistoceno) e sobre a história dos ambientes marginas registrados nessas unidades na Ilha Seymour, Antártica Ocidental, foram propostos os seguintes objetivos:

1. Investigar a taxonomia, a tafonomia e a paleoecologia dos foraminíferos, que são os restos mais abundantes e diversos nos depósitos, e também dos outros microfósseis com paredes inorgânicas (i.e., mineralizadas) associados.

2. Definir a distribuição estratigráfica dos microfósseis nas seções analisadas e, com base em fósseis-índice, refinar o conhecimento sobre as idades das formações.

3. Investigar a distribuição geográfica dos táxons de foraminíferos encontrados, comparando-os com aqueles de localidades próximas e de outros continentes e províncias biogeográficas pretéritas e atuais.

4. Elaborar e testar hipóteses quanto aos contextos deposicionais dos depósitos, bem como sobre suas possíveis relações com mudanças nas assembleias fossilíferas. 

7. CONCLUSÃO 



\subsection{Paleobiologia das assembleias}

Os foraminíferos da parte superior da Formação La Meseta são raros, mas a presença das espécies Textularia sp. 1 e Textularia sp. 2 como restos autóctones ou parautóctones evidenciam, ao menos em parte, a composição das comunidades infaunais que prosperavam na área. $O$ gênero Textularia não ocorre nas assembleias alóctones da parte inferior da unidade, compostas quase exclusivamente por formas calcárias epifaunais e infaunais. Isto pode ser explicado pelo fato das formas aglutinadas serem facilmente obliteradas por processos tafonômicos físicos, como transporte e compactação, enquanto as formas calcárias são mais resistentes - embora sejam muito suscetíveis à dissolução

A associação, em algumas assembleias das formações Hobbs Glacier e Weddell Sea, de espécimes pequenos ou diminutos de foraminíferos calcários infaunais e planctônicos e de espécimes grandes de foraminíferos aglutinados epifaunais impôs algumas dificuldades para a interpretação da história tafonômica e da paleoecologia das assembleias. Todavia, essas questões foram dirimidas pela identificação de feições tafonômicas exclusivas dos grandes foraminíferos aglutinados, que evidenciaram sua reelaboração a partir de depósitos mais antigos, e também pela identificação taxonômica, que revelou que a maioria de seus táxons são típicos ou exclusivos de ambientes marinhos profundos, antigos ou modernos.

As assembleias - principalmente aquelas encontradas na seção Sey-03, onde os restos são mais abundantes e bem preservados - indicam a presença de antigas comunidades bentônicas infaunais tanto na Formação Hobbs Glacier quanto na Formação Weddell Sea. Na Formação Hobbs Glacier, as evidências destas comunidades são encontradas nas assembleias Sey-03-07, representadas pelos espécimes do foraminífero lagenído Oolina stellula, que ocorrem associados aos espécimes contemporâneos do foraminífero planctônico Neogloboquadrina dutertrei e do radiolário Larcopyle polyacantha. Já na Formação Weddell Sea, estas evidências correspondem aos espécimes do foraminífero rotalíido Globocassidulina subglobosa da assembleia Sey-03-03, que ocorrem associados aos raros mas bem preservados espécimes da diatomácea ?Coscinodiscus sp., e aos espécimes do lagenído Favulina hexagona da assembleia Sey-03-01, que ocorrem associados ao foraminífero planctônico Globigerinita uvula.

Além do estado inalterado e das quantidades relativamente grandes destes espécimes, a presença de indivíduos de diferentes estágios ontogenéticos (i.e., de diferentes tamanhos e/ou com 
diferentes números de câmaras) indicam que os restos são ao menos parcialmente representativos das comunidades que se desenvolviam na área, uma vez que suas populações obrigatoriamente seriam formadas por indivíduos de uma mesma geração, ou de gerações próximas, em diferentes estágios ontogenéticos.

Nas mesmas assembleias (Sey-03-07, Sey-03-03 e Sey-03-01) também são encontrados foraminíferos aglutinados relativamente grandes e de gêneros e espécies típicas ou exclusivas de assembleias do tipo flysch, como Ammodiscus spp., Bathysiphon sp, Cyclammina placenta, Nothia robusta, Reticulophragmium garcilassoi, Recurvoides sp., Saccammina grzybowski e Sculptobaculites barri. Todavia, embora estas formas sejam mais comuns nas assembleias já citadas, algumas também são encontradas em assembleias de outros níveis de ambas as unidades (i.e., Sey-10-17, Sey-13-69 e Sey-14-07, Formação Hobbs Glacier; Sey-07-01, Formação Weddell Sea), como Ammomarginulina cf. auberta, Budashevaella cf. laevigata, Cyclammina cancellata e Glomospira charoides.

Os táxons citados acima, especialmente aqueles encontrados nas assembleias Sey-03-07 e Sey-03-01, sugerem fortemente que as formas aglutinadas ocorriam associadas em assembleias do tipo flysch de rochas mais antigas, das quais foram exumadas e reelaboradas nos diamictitos das formações Hobbs Glacier e Weddell Sea, uma vez que todos os espécimes apresentam as mesmas feições tafonômicas, como preenchimento interno por micrito litificado e cobertura superficial pelo mesmo material, além de ocasional fragmentação posterior. Isto também é sugerido para as outras assembleias, embora em algumas delas essas formas sejam mais raras e menos diversas.

Por fim, alguns destes táxons aglutinados ocorrem associados em depósitos paleocênicos do sudoeste do Pacífico, no Mar da Tasmânia e nas proximidades da Nova Zelândia e da Nova Guiné. É especial a ocorrência de $S$. grzybowski, que possui espécimes virtualmente idênticos no Argilito Moogli (Paleoceno) da Nova Guiné e na Formação Weddell Sea na Ilha Seymour, os quais diferem significativamente de $S$. grzybowski de outras localidades. A melhor explicação para tal similaridade é que os espécimes representam a mesma população ou populações próximas, o que é coerente com a paleogeografia do Paleoceno e com a ocorrência de $R$. garcilassoi, um fóssilíndice desta época e que também ocorre no sudoeste do Oceano Pacífico. 


\subsection{Paleoambientes das formações Hobbs Glacier e Weddell Sea}

O contexto deposicional glacio-marinho da Formação Hobbs Glacier já fora estabelecido pela presença de estratificações cruzadas assimétricas em diversos níveis, indicando a presença de uma corrente fluindo em direção ao nordeste ou leste durante toda a deposição da unidade, e pela presença de fósseis bem preservados de animais exclusivamente marinhos, como briozoários e braquiópodes. De qualquer forma, esta interpretação é reforçada pelas assembleias aqui relatadas, com restos autóctones ou parautóctones de foraminíferos bentônicos, escleritos bem preservados de poríferos hexactinelídeos e foraminíferos planctônicos.

As assembleias de microfósseis com paredes inorgânicas, com espécimes autóctones ou parautóctones associados a espécimes reelaborados, também apresentam o mesmo padrão de mistura espacial e temporal observado nas assembleias de palinomorfos dos estratos da Formação Hobbs Glacier da Ilha James Ross, nas quais palinomorfos cretácicos e paleocênicos ocorrem associados a formas miocênicas contemporâneas à deposição da unidade.

Por outro lado, o contexto deposicional da Formação Weddell Sea, interpretado na descrição formal da unidade como plenamente glacial, se revelou glacio-marinho ao menos para determinados estratos, tendo em vista a presença de foraminíferos bentônicos autóctones ou parautóctones, de foraminíferos planctônicos contemporâneos à deposição, de um fragmento bem preservado de colônia ereta de briozoários e, principalmente, de abundantes moldes calcários de icnofósseis na assembleia Sey-07-02, os quais indicam a presença de animais vermiformes bioturbadores cujos vestígios só puderam ser extraídos da rocha por serem compostos por cristais de carbonato de cálcio intricados e relativamente robustos.

De fato, outros autores já haviam proposto um contexto glacio-marinho para a Formação Weddell Sea, mas se basearam apenas na interpretação de que os clastos presentes na unidade representavam clastos caídos. Assim, embora uma colônia de briozoários incrustantes tenha sido relatada na própria descrição formal da unidade - sem ter sido considerada como um sinal de influência marinha -, as assembleias aqui relatadas representam as primeiras evidências paleontológicas robustas para a definição de um contexto glacio-marinho para a Formação Weddell Sea. 



\subsection{IdAde DAS FORMAÇões HobbS GLACIER E WEDDELl SEA}

Dos táxons encontrados, raros são considerados fósseis-índice, o que impediu um maior refinamento da idade conhecida para as formações. Alguns deles, no entanto, corroboraram ou impuseram novas idades mínimas para as unidades.

A presença de Reticulophragmium garcilassoi, um fóssil-índice para o Paleoceno, na Formação Hobbs Glacier indica que os espécimes foram reelaborados de depósitos paleocênicos, os quais não podem ser correlacionados àqueles das formações López de Bertodano e Sobral, uma vez que $R$. garcilassoi não ocorre nestes depósitos - da mesma forma como a maioria dos outros táxons encontrados. E, de fato, nenhuma outra unidade da Península Antártica pode ser proposta como fonte destes restos. Além disso, há espécimes de $R$. garcilassoi de diferentes estágios ontogenéticos, o que atesta que a tafocenose original representava ao menos parcialmente à biocenose e que os restos não sofreram muito retrabalhamento após terem sido exumados de um depósito indeterminado e reinseridos na Formação Hobbs Glacier. Portanto, é provável que o depósito original, a partir do qual foram reelaborados, se encontrava próximo à área de deposição da Formação Hobbs Glacier, ou então que clastos destes depósitos, contendo R. garcilassoi, foram trazidos de áreas mais distantes, provavelmente pela ação de geleiras.

Para a Formação Hobbs Glacier, cuja idade miocênica foi estabelecida pela comparação entre a curva isotópica de oxigênio global e os dados obtidos a partir de carapaças de cirripédios, com uma idade mínima miocênica indicada pela presença do briozoário Microporella, a presença do foraminífero Neogloboquadrina dutertrei e do radiolário Larcopyle polyacantha - cujas distribuições cronoestratigráficas abrangem do Mioceno Superior ao Recente e do Mioceno Inferior ao Plioceno Inferior, respectivamente - impõe a mesma restrição à idade mínima.

Para a Formação Weddell Sea, cuja datação é bem menos precisa, com idade estimada entre o Plioceno Inicial e o Pleistoceno, a descoberta de espécimes do foraminífero planctônico Globigerinita uvula não permite inferir novas restrições de idade. Entretanto, uma vez que a espécie não é encontrada nos depósitos cenozoicos da Península Antártica, mas naqueles neogênicos e quaternários da porção sul do Platô Kerguelen - extremo sul do Oceano Índico, próximo ao contato com o Oceano Austral -, estudos posteriores podem revelar possíveis correlações cronológicas entre os depósitos. 



\section{REFERÊNCIAS BIBLIOGRÁFICAS}



Akers, W. H. 1954. Ecological aspects and stratigraphic significance of the foraminifera Cyclammina cancellata Brady. Journal of Paleontology, v. 28, pp. 132-152.

Altenbach, A. V.; Lutze, G. F.; Schiebel, R.; Schönfeld, J. 2003. Impact of interrelated and interdependent ecological controls on benthic foraminifera: An example from the Gulf of Guinea. Palaeogeography, Palaeoclimatology, Palaeoecology, v.197, n. 3-4, pp. 213-238.

Armstrong, H.; Brasier, M. D. 2005. Microfossils. 2a ed. Oxford: Blackwell Publishing.

Arnold, A. J.; Parker, W. C. 1999. Biogeography of planktonic foraminifera. In: Sen Gupta, B. K. (Ed.). Modern foraminifera. Dordrecht: Kluwer Academic Publishers, pp. 103-121.

Bandy, O. L.; Echols, R. J. 1964. Antarctic foraminiferal zonation. Antarctic Research Series, v. 1, pp. 73-91.

Barke, J.; Burgh, J.; Konijnenburg-van Cittert, J. H. A.; Collinson, M. E.; Pearce, M. A.; Bujak, J.; Heilmann-Clausen, C.; Speelman, E. N.; Kempen, M. M. L.; Reichart, G. J; Lotter, A. F.; Brinkhuis, H. 2012. Coeval Eocene blooms of the freshwater fern Azolla in and around Arctic and Nordic seas. Palaeogeography, Palaeoclimatology, Palaeoecology, v. 337-338, pp. 108-119.

Barret, P. J. 2009. A history of Antarctic Cenozoic glaciation. In: Florindo, F.; Siegert, M. Antarctic climate evolution. Amsterdam: Elsevier, pp. 33-83.

Barron, J.; Larsen, B. (Eds.). 1989. Proceedings of the ODP leg 119: Initial reports. Texas: College Station.

Bartoli, G.; Sarnthein, M.; Weinelt, M.; Erlenkeuser, H.; Garbe-Schonberg, D. 2005. Final closure of Panama and the onset of northern hemisphere glaciation. Earth and Planetary Science Letters, v. 237 , n. $1-2$, p. 33-44.

Bé, A. W. H. 1968. Zoogeography of Antarctic and Subantarctic planktonic foraminifera in the Atlantic and Pacific ocean sectors. Antarctic Journal, v. 2, n. 5, pp. 188-189.

Bé, A. W. H. 1977. An ecological, zoogeographical and taxonomic review of recent planktonic foraminifera. In: Ramsay, A. T. S. (Ed.). Oceanic micropaleontology. London: Academic Press, pp. $1-100$.

Begg, J. G.; Cave, M. P.; Campbell, J. D. 1983. Terebellina mackay Bather in the South Island. New Zealand Journal of Geology and Geophysics, v. 26, pp. 121-122.

Beldean, C.; Filipescu, S. 2011. "Flysch-type" agglutinated foraminifera from the Lower Miocene of the Transylvanian Basin (Romania). In: Kaminski, M. A.; Filipescu, S. (Eds.). Proceedings of the Eighth International Workshop on Agglutinated Foraminifera. Grzybowski Foundation Special Publication, v. 16, pp. 1-18. 
Bernhard, J. M.; Sen Gupta, B. K. 1999. Foraminifera of oxygen-depleted environments. In: Sen Gupta, B. K. (Ed.). Modern foraminifera. Dordrecht: Kluwer Academic Publishers, pp. 201-216.

Berggren, W. A.; Kaminski, M. A. 1990. Abyssal agglutinates: Back to basics. In: Hemleben, C.; Kaminski, M. A.; Kuhnt, W.; Scott, D. B. (Eds.). Paleoecology, biostratigraphy, paleoceanography and taxonomy of agglutinated foraminifera. Massachussets: Kluwer Academic Publishers, pp. 53-76.

Berkeley, A.; Perry, C. T.; Smithers, S. G.; Horton, B. P.; Taylor, K. G. 2007. A review of the ecological and taphonomic controls on foraminiferal assemblage development in intertidal environments. Earth-Science Reviews, v. 83, pp. 205-230.

Bijl, P. K.; Houben, A. J. P.; Schouten, S.; Bohaty, S. M.; Sluijs, A.; Reichart, G. J.; Sinninghe Damste, J. S.; Brinkhuis, H. 2010. Transient Middle Eocene atmospheric $\mathrm{CO}_{2}$ and temperature variations. Science, v. 330, pp. 819-821

Bijl, P. K.; Schouten, S.; Sluijs, A.; Reichart, G. J.; Zachos, J. C.; Brinkhuis, H. 2009. Early Paleogene temperature evolution of the southwest Pacific Ocean. Nature, v. 461, pp. 776-779.

Birkenmajer, K. 1980. Discovery of Pliocene glaciation on the King George Island, South Shetlands Islands (West Antarctica). Bulletin of the Polish Academy of Sciences, v. 27, p. 59-67.

Birkenmajer, K.; Gazdzicki, A.; Wrona, R. 1983. Cretaceous and Tertiary fossils in glacio-marine strata at Cape Melville, Antarctica. Nature, v. 303, pp. 56-59.

Birkenmajer, K.; Luczkowska, E. 1987. Foraminiferal evidence for a Lower Miocene age of glaciomarine and related strata, Moby Dick Group, King George Island (South Shetlands Islands, Antarctica). Studia Geologica Polonica, v. 90, pp. 81-123.

Bitner, M. A. 1996. Encrusters and borers of brachiopods from the La Meseta Formation (Eocene) of Seymour Island, Antarctica. Polish Polar Research, v. 17, n. 1-2, pp. 21-28.

Boardman, R. S.; Cheetham, A.; Rowell, A. J. (Eds.). 1987. Fossil invertebrates. Palo Alto: Blackwell Scientific Publication.

Boardman, R. S.; Cheetham, A. H. 1987. Phylum Bryozoa. In: Boardman, R. S.; Cheetham, A.; Rowell, A. J. (Eds.). Fossil invertebrates. Palo Alto: Blackwell Scientific Publication, pp. 497549.

Bohaty, S. M.; Zachos, J. C. 2003. Significant Southern Ocean warming event in the late Middle Eocene. Geology, v. 31, n. 11, pp. 1017-1020.

Bolli, H. M.; Saunders, J. B. 1989. Oligocene to Holocene low latitude planktic foraminifera. In Bolli, H. M.; Saunders, J. B.; Perch-Nielsen, K. (Eds.). Plankton Stratigraphy. Cambridge: Cambridge University Press, v. 1, pp. 165-262. 
Boltovskoy, E.; Giussani, G.; Watanabe, S.; Wright, R. C. 1980. Atlas of benthic shelfforaminifera on the southwest Atlantic. Boston: Dr. W. Junk.

BouDagher-Fadel, M. K. 2013. Biostratigraphic and geological significance of planktonic foraminifera. 2a ed. London: UCL Press.

Brady, H. B. 1884. Report on the foraminifera dredged by H. M. S. Challenger during the years 1873-1876. In: Murray, J. (Ed.). Reports of the scientific results of the voyage of the H.M.S.

Challenger, v. 9 (Zoology), pp. 1-814.

Breza, J.; Wise, S. W. 1992. Lower Oligocene ice-rafted debris on the Kerguelen Plateau: Evidence for East Antarctic continental glaciation. Proceedings of the Ocean Drilling Program, Scientific Results, v. 120, pp. 161-178.

Brinkhuis, H.; Schouten, S. 2006. Episodic fresh surface waters in the Eocene Arctic Ocean. Nature, v. 441, n. 7093, pp. 606-609.

Brood, K. 1998. Bryozoa. In: Haq, B. U.; Boersma, A. (Eds.). Introduction to marine micropaleontology. 2a ed. Amsterdam: Elsevier, pp. 189-201.

Brouwer, J. 1965. Agglutinated foraminiferal faunas from some turbiditic sequences I, II. Proceedings of the Koninklijke Nederlandse Akademie van Wetenschappen series B, v. 68, pp. 309-334.

Brown, B.; Gaina, C.; Müller, R. D. 2006. Circum-Antarctic palaeobathymetry: Illustrated examples from Cenozoic to recent times. Palaeogeography, Palaeoclimatology, Palaeoecology, v. 231, pp. 158-168.

Brusca, R. C.; Brusca, G. J. 2007. Invertebrados. 2a ed. Rio de Janeiro: Guanabara Koogan.

Bubík, M. 2009. Nové a málo známé aglutinované foraminifery z paleogé nu jižní Moravy. Zprávy o geologických výzkumech, v. 12, p. 82-85.

Cabrera, M. I. L.; Olivero, E. B. 2011. An Eocene articulated Polyplacophora (Mollusca) from the La Meseta Formation, Antarctica, and the stratigraphy of the fossil-bearing strata. Journal of Paleontology, v. 85, n. 5, pp. 970-976.

Campbell, J. K.; Campbell, J. D. 1969. Triassic tube fossils from Tupaeka rocks, Akatore, South Otago. New Zealand journal of Geology and Geophysics, v. 13, pp. 392-399.

Cantrill, D. J.; Poole, I. 2012. The vegetation of Antarctica through geological time. Cambridge: Cambridge University Press.

Caramés, A.; Concheyro, A. 2013. Late Cenozoic foraminifera from diamictites of Cape Lamb, Vega Island, Antarctic Peninsula. Ameghiniana, v. 50, n. 1, pp. 114-135. 
Carpenter, W. B.; Parker, W. K.; Jones, T. R. 1862. Introduction to the study of the foraminifera. London: Ray Society.

Chaisson, W. P.; Leckie, R. M. 1993. High-resolution planktonic foraminifer biostratigraphy of site 806, Ontong River Plateau (Western Equatorial Pacific). In: Kroenke, W. H.; Mayer, L. W. (Eds.). Proceedings of the Ocean Drilling Program, Scientific results, pp. 137-178.

Charnock, M. A.; Jones, R. W. 1990. Agglutinated foraminifera from the Palaeogene of the North Sea. In: Hemleben, C.; Kaminski, M. A.; Kuhnt, W.; Scott, D. B. (Eds.). Paleoecology, biostratigraphy, paleoceanography and taxonomy of agglutinated foraminifera. Kluwer Academic Publications, pp. 139-244.

Chen, P. H. 1975. Antarctic radiolaria. Initial Reports of the Deep Sea Drilling Project, v. 28, pp. 437-513.

Chukwu, J. N.; Kosun, E. A.; Alkali, Y. B. 2012. Foraminiferal biostratigraphy and depositional environment of Oloibiri-1 Well, eastern Niger Delta, Nigeria. Journal of Geography and Geology, v. 4, n. 4, pp. 114-122.

Clark, D. L. 1990. Stability of the Arctic Ocean ice-cover and Pleistocene warming events: Outlining the problem. In: Bleil, U.; Thiede, J. (Ed.). Geological history of the polar oceans: Arctic versus Antarctic. London: Springer, pp. 279-288.

Concheyro, A.; Salani, F. M.; Adamonis, S.; Lirio, J. M. 2007. Los depósitos diamictíticos cenozoicos de la cuenca James Ross, Antártida: una síntesis estratigráfica y nuevos hallazgos paleontológicos. Revista de la Asociación Geológica Argentina, v. 62, n. 4, pp. 568-585.

Concheyro, A.; Caramés, A.; Amenábar, C. R.; Lescano, M. 2014. Nannofossils, foraminifera and microforaminiferal linings in the Cenozoic diamictites of Cape Lamb, Vega Island, Antarctica. Polish Polar Research, v. 35, n. 1, pp. 1-26.

Corliss, B. H. 1991. Morphology and microhabitat preferences of benthic foraminifera from the northwest Atlantic Ocean. Marine Micropaleontology, v. 17, p. 195-236.

Cornelius, N.; Gooday, A. J. 2004. 'Live'(stained) deep-sea benthic foraminiferans in the western Weddell Sea: Trends in abundance, diversity, and taxonomic composition along a depth transect. Deep-Sea Research II, v. 51, pp. 1571-1602.

Cortese, G.; Zarikian, C. A. A. 2015. Data report: Radiolaran occurrences at IODP Expedition 329 Site U1317. Proceedings of the Integrated Ocean Drilling Project, v. 329, pp. 1-13.

Craddock, C. (Ed.). 1982. Sedimentology and palaeocurrent analysis of the basal part of the Beacon Supergroup (Devonian (and older?) to Triassic) in south Victoria Land, Antarctica. Madison: University of Wisconsin Press. 
Culver, S. J. 1993. Foraminifera. In: Lipps, J. H. (Ed.). Fossil prokaryotes and protists. Oxford: Blackwell Scientific Publications, pp. 203-247.

Culver, S. J.; Buzas, M. A. 1982. Distribution of Recent benthic foraminifera in the Caribbean region. Washington: Smithsonian Institution Press.

Cushman, J. A. 1948. Foraminifera: Their classification and economic use. 4a ed. Cambridge: Harvard University Press.

Cusminsky, G. C.; Whatley, R. C. 2008. Calcareous microfossils (foraminifera and ostracoda) of the Late Cenozoic from Patagonia and Tierra del Fuego: A review. In: Rabassa, J. (Ed.). The Late Cenozoic of Patagonia and Tierra del Fuego. Amsterdam: Elsevier, pp. 327-342.

Davies, B. 2014. James Ross Island project. Disponível em: http://www.antarcticglaciers.org /about-2/bethan-davies-2/our-research-and-team/jamesrossisland/. Acessado em 12 de fevereiro de 2014.

Debenay, J. P. 2012. A guide to 1,000 foraminifera from southwestern Pacific New Calenonia. Paris: Muséum National d'Histoire Naturelle.

DeConto, R. M.; Pollard, D. 2003. Rapid Cenozoic glaciation of Antarctica induced by declining atmospheric $\mathrm{CO}_{2}$. Nature, v. 421, pp. 245-249.

Del Valle, R. A.; Elliot, D. H.; Macdonald, D. I. M. 1992. Sedimentary basins on the east flank of the Antarctic Peninsula: Proposed nomenclature. Antarctic Science, v. 4, n. 4, pp. 477-478.

Dietze, V.; Cresta, S.; Martire, L.; Pavia, G. 2017. Ammonites, taphonomical analysis and biostratigraphy of the Aalenian (Middle Jurassic) from Capo San Vigilio (Lake Garda, N Italy). Neues Jahrbuch für Geologie und Paläontologie, v. 284, n. 2, pp. 161-206.

Dingle, R. V.; McArthur, J. M.; Vroon, P. 1997. Oligocene and Pliocene interglacial events in the Antarctic Peninsula dated using strontium isotope stratigraphy. Journal of the Geological Society, v. 154 , n. 2, pp. 257-264.

Dingle, R. V.; Lavelle, M. 1998. Antarctic peninsular cryosphere. Early Oligocene (c. 30 M) initiation and a revised glacial chronology. Journal of the Geological Society, v. 155, pp. 422-437.

d'Orbigny, A. 1826. Tableau méthodique de la classe des Céphalopodes. Annales des Sciences Naturelles, v. 7, pp. 245-314.

Dunham, R. J. 1962. Classification of carbonate rocks according to depositional textures. American Association of Petroleum Geologists, v. 1, pp. 108-121.

Eichwald, C. E. 1830. Zoologia Specialis. Eichwaldus, Vilnae. 
Elliot, D. H. 1988. Tectonic setting and evolution of the James Ross Basin, northern Antarctic Peninsula. Geological Society of America Memoirs, v. 169, pp. 541-556.

Elliot, D. H.; Rinaldi, C.; Zinsmeister, W. J.; Trautman, T. A.; Bryant, W. A.; Del Valle, R. 1975. Geological investigations on Seymour Islands, Antarctic Peninsula. Antarctic Journal, v. 10, n. 4, pp. 182-186.

Elliot, D. H.; Trautman, T. A. 1977. Lower Tertiary strata on Seymour Island, Antarctic Peninsula. $3^{\text {rd }}$ Symposium on Antarctic Geology and Geophysics. Madison, Wisconsin. Abstracts, p. 168.

Elliot, D. H.; Trautman, T. A. 1982. Lower Tertiary strata on Seymour Island, Antarctic Peninsula. In: Craddock, C. (Ed.). Antarctic Geoscience. Madison: University of Wisconsin Press, pp. 287297.

Elliot, N. F.; Davies, B. J.; Carrivick, J. L.; Rodés, A.; Hambrey, M. J.; Smellie, J. L.; Domak, E. 2014. Ice-stream initiation, duration and thinning on James Ross Island, northern Antarctic Peninsula. Quaternary Science Reviews, v. 86, n. 15, pp. 78-88.

Ellis, B. F.; Messina, A. R. 1940. Catalogue of foraminifera. New York: American Museum of Natural History.

Evitt, W. R. 1963. A discussion and proposals concerning fossil dinoflagellates, hystrichospheres and acritarchs. Proceedings of the National Academy of Sciences, v. 49, pp. 158-164.

Fedorov, A. V.; Dekens, P. S.; McCarthy, M.; Ravelo, A. C.; Menocal, P. B.; Barreiro, M.; Pacanowski, R. C.; Philander, S. G. The Pliocene paradox: Mechanisms for a permanent El Niño. Science, v. 312, p. 1485-1489.

Feldmann, R. M.; Gazdzicki, A. 1977. A new species of Glyphae (Decapoda: Palinura) from the La Meseta Formation (Eocene) of Seymour Island, Antarctica. Acta Palaeontologica Polonica, v. 42, n. 3, pp. 437-445.

Fernández-López, S. R.; Henriques, M. H.; Linares, A.; Sandoval, J; Ureta, M. S. 1999. Aalenian Tmetoceras (Ammonoidea) from Iberia: Taphonomy and paleobiogeography. In: Olóriz, F.; Rodriguez-Tovar, F. J. (Eds.). Advancing research on living and fossil cephalopods. New York: Kluwer Academic/Plenum Publishers, pp. 395-418.

Filipescu, S.; Beldean, C. 2008. Foraminifera in the deep-sea environments of the lower Hida Formation (Transylvanian Basin, Romania). Acta Palaeontologica Romaniae, v. 6, pp. 105-114.

Flugel, E. 2004. Microfacies of carbonate rocks: Analysis, interpretation and application. 2a ed. Heilderberg: Springer.

Foster, G. L.; Lear, C. H.; Rae, J. W. B. 2012. The evolution of pCOs, ice volume and climate during the Middle Miocene. Earth and Planetary Science Letters, v. 341-344, pp. 243-254. 
Francis, J. E.; Marenssi, S.; Levy, R.; Hambrey, M.; Thorn, V. T.; Mohr, B.; Brinkhius, H.; Warnaar, J.; Zachos, J.; Bohaty, S.; DeConto, R. 2009. From greenhouse to icehouse: The Eocene/Oligocene in Antarctica. In: Florindo, F.; Siegert, M. (Eds.). Antarctic climate evolution. Amsterdam: Elsevier, pp. 309-368.

Fuente, M. S.; Santillana, S. N.; Marenssi, S. A. 1995. An Eocene leatherback turtle (Cryptodira: Dermochelyidae) from Seymour Island, Antarctica. Stvdia Geologica Salmanticensia, v. 31, pp. 21-34.

Futterer, D. K. 1984. Pithonelloid calcareous dinoflagellates from the Upper Cretaceous and Cenozoic of the Southeastern Atlantic Ocean, Deep Sea Drilling Project Leg $74^{1}$. In: Moore, T. C., Jr.; Rabinowitz, P. D. (Eds.) Initial reports of the DSDP, v. 74. Washington: U.S. Government Printing Office, pp. 533-541.

Gage, J. D.; Tyler, P. A. 1999. Deep-sea biology: A natural history of the organisms at the deepsea floor. Cambridge: Cambridge University Press.

Galazzo, F. B.; Thomas, E.; Pagani, M.; Warren, C.; Luciani, V.; Giusberti, L. 2014. The Middle Eocene Climatic Optimum (MECO): A multiproxy recordo f paleoceanographic changes in the southeast Atlantic (ODP Site 1263, Walvis Ridge). Paleoceanography, v. 29, n. 12, pp. 11431161.

Galloway, J. J. 1933. A manual of foraminifera. Bloomington: The Principia Press.

Gazdzicka, E.; Gazdzicki, A. 1994. Recycled Upper Cretaceous calcareous nannoplankton from the Pecten Conglomerate of Cockburn Island, Antarctica. Polish Polar Research, v. 15, pp. 3-13.

Gazdzicki, A. 1989. Planktonic foraminifera from the Oligocene Polonez cove Formation of King George Island, West Antarctica. Polish Polar Research, v. 10, n. 1, pp. 47-55.

Gazdzicki, A. 1993. Geology and paleobiology of Cockburn Island, Antarctic Peninsula. In: XX Polar Symposium, Lublin. Abstracts, pp. 329-332.

Gazdzicki, A.; Majewski, W. 2012. Foraminifera from the Eocene La Meseta Formation of Isla Marambio (Seymour island), Antarctic Peninsula. Antarctic Science, v. 24, n. 4, pp. 408-416.

Gazdzicki, A.; Tatur, A.; Hara, U.; Del Valle, R. A. 2004. The Weddell Sea Formation: Post-Late Pliocene terrestrial glacial deposits on Seymour Island, Antarctic Peninsula. Polish polar Research, v. 25, n. 3-4, pp. 189-204.

Gazdzicki, A.; Webb, P. N. 1996. Foraminifera from the Pecten conglomerate (Pliocene) of Cockburn island, Antarctic Peninsula. Palaeontologia Polonica, n. 55, pp. 147-174.

Gelfo, J. N.; Mörs, T.; Lorente, M.; López, G. M. 2015. The oldest mammals from Antarctic, early Eocene of the La Meseta formation, Seymour Island. Palaeontology, v. 58, n. 1, pp. 101-110. 
Gleason, J. D.; Thomas, D. J.; Moore, T. C., Jr.; Blum, J. D.; Owen, R. M.; Haley, B. A. 2009. Early to middle Eocene history of the Arctic Ocean from $\mathrm{Nd}-\mathrm{Sr}$ isotopes in fossil fish debris, Lomonosov Ridge. Paleoceanography, v. 24, n. 2, pp. 1-18.

Goldner, A.; Herold, N.; Huber, M. 2014. The challenge of simulating the warmth of the midMiocene climatic optimum in CESM1. Climate of the Past, v. 10, pp. 523-536.

Goldstein, S. T. 1999. Foraminifera: A biological overview. In: Sen Gupta, B. K. (Ed.) Modern foraminifera. Dordrecht: Kluwer Academic Publishers, pp. 37-55.

Gooday, A. J.; Bowser, S. S.; Bernhard, J. M. 1996. Benthic foraminiferal assemblages in Explorers Cove, Antarctica: A shallow-water site with deep-sea characteristics. Progress in Oceanography, v. 37, n. 2, pp. 117-166.

Gradstein, F. M.; Berggren, W. A. 1981. Flysch-type agglutinated foraminifera and the Maestrichtian to Paleogene history of the Labrador and North seas. Marine Micropaleontology, v. 6, pp. 211-269.

Green, O. R. 2001. A manual of practical laboratory and field techniques in palaeobiology. New York: Springer.

Gross, O. 2001. Foraminifera. In: Costello, M. J. et al. (Eds.). European register of marine species: A check-list of the marine species in Europe and a bibliography of guides to their identification. Collection Patrimoines Naturels, v. 50, pp. 60-75

Hambrey, M. J.; Smellie, J. L. 2006. Distribution, lithofacies and environmental context of Neogene glacial sequences on James Ross and Vega islands, Antarctic Peninsula. In: Francis, J. E.; Pirrie, D.; Crame, A. (Eds.). Cretaceous-Tertiary high-latitude palaeoenvironments, James Ross Basin, Antarctic Peninsula region. London: Geological Society of London, pp. 187-200.

Hamilton, W.; Hayes, P. T. 1963. Type section of the Beacon Sandstone of Antarctica. Geological Survey Professional Paper, v. 456-A, pp. 1-18.

Harloff, J; Mackensen, A. 1997. Recent benthic foraminiferal associations and ecology of the Scotia Sea and Argentine Basin. Marine Micropaleontology, v. 3, n. 1-2, pp. 1-29.

Hart, 1988. Early Miocene agglutinated foraminifera from the Bermuda Abyssal Plain: DSDP Site 603 (NW Atlantic Ocean). Abh. Geol. B.-A., v. 41, pp. 121-131.

Hathway, B. 2000. Continental rift to back-arc basin: Jurassic-Cretaceous stratigraphical and structural evolution of the Larsen Basin, Antarctic Peninsula. Journal of the Geological Society, London, v. 157, pp. 417-432.

Hathway, B.; Riding, J. B. 2001. Stratigraphy and age of the Lower Cretaceous Pedersen Formation, northern Antarctic Peninsula. Antarctic Science, v. 13, n. 1, pp. 67-74. 
Hatta, A. 2013. The foraminifera of the Chuuk Lagoon, and its value as teaching material. Occasional Papers, n. 53, pp. 1-5.

Haywood, A. M.; Dowsett, H. J.; Valdes, P. L.; Lunt, D. J.; Francis, J. E.; Sellwood, B. W. 2009. Pliocene climate, process and problems. Philosophical Transactions of the Royal Society A, v. 367, pp. 3-17.

Hayward, B. W.; Holzmann, M.; Grenfell, H. R. 2004. Morphological distinction of molecular types in Ammonia: Towards a taxonomic revision on the world's most common and misidentified foraminiferal genus. Marine Micropaleontology, v. 50, pp. 237-271.

Hemleben C.; Spindler, M.; Anderson, O. R. 1989. Modern planktonic foraminifera. New York: Springer-Verlag.

Henning, A. 1910. Le conglomérat Pleistocène à Pecten de l'ile Cockburn. Wissenschaftliche Ergebnisse der Schwedischen Südpolar-Expedition, 1901-03, Geologie und Paläontologie, v. 3, n. 10, pp. 1-73.

Herb, R 1968. Recent planktonic foraminifera from sediments of the Drake Passage, Southern Ocean. Ecologae Geologicae Helvetiae, v. 61, n. 2, pp. 467-480.

Herrero, C.; Canales, M. L. 2002. Taphonomic processes in selected Lower and Middle Jurassic foraminifera from the Iberian range and Basque-Cantabrian Basin (Spain). Journal of Foraminiferal Research, v. 32, n. 1, pp. 22-42.

Hilbrecht, H. 1997. Morphologic gradation and ecology in Neogloboquadrina pachyderma and $N$. dutertrei (planktic foraminifera) from core top sediments. Marine Micropaleontology, v. 31, pp. 31-43.

Holland, R. 1910. The fossil foraminifera. Wissenschaftliche Ergebnisse der Schwedischen Südpolar-Expedition, 1901-03, Geologie und Paläontologie, v. 3, n. 9, pp. 1-12.

Hollande, A.; Enjumet, M. 1960. Cytologie, évolution et systématique des Sphaeroidés (Radiolaires). Archives du Muséum National d'Histoire Naturelle, v. 7, pp. 1-134.

Holbourn, A. E.; Henderson, A. S. 2002. Re-illustration and revised taxonomy for selected deepsea benthic foraminifers. Palaeontologia Electronica, v. 4, n. 2, pp. 1-34.

Holbourn, A. E.; Henderson, A. S.; MacLeod, N. 2013. Atlas of benthic foraminifera. Oxford: Natural History Museum/Wiley-Blackwell.

Holz, M.; Simões, M. G. 2002. Elementos fundamentais de tafonomia. Porto Alegre: Editora da UFRGS.

Hooper, J. N. A.; Soest, R. W. M. (Eds.). 2013. Systema Porifera: A guide to the classification of sponges. 2 vols. Heilderberg: Springuer. 
Hottinger, L.; Scheuring, V. 1997. Glossary of terms used in foramininferal research. Disponível em: http://www.ucmp.berkeley.edu/people/jlipps/glossary.html. Acessado em 20 de janeiro de 2016.

Hsü, K. J. 1984. A scenario for the terminal Cretaceous event. Initial Reports of the Deep Sea Drilling Project, v. 13, pp. 755-763.

Huber, B. T. 1986. Foraminiferal distribution across the Cetaceous/Tertiary transition on Seymour Island, Antarctic Peninsula. Antarctic Journal of the United States, v. 21, n. 5, pp. 71-73.

Huber, B. T. 1988. Upper Campanian-Paleocene foraminifera from the James Ross Island region, Antarctic Peninsula. p. 163-252. In: Feldmann, R. M.; Woodburne, M. O. (Eds.). Geology and Paleontology of Seymour Island, Antarctic Peninsula. Geological Society of America, Memoir 169, pp. 163-252.

Huber, B. T. 1991. Paleogene and Early Neogene planktonic foraminifer biostratigraphy of sites 738 and 744, Kerguelen Plateau (Southern Indian Ocean). In: Barron, J; Larsen, B. (Eds.). Proceedings of the Ocean Drilling Program, Scientific Results, College Station, TX (Ocean Drilling Program), v. 119, p. 427-449.

Huber, M.; Nof, D. 2006. The ocean circulation in the southern hemisphere and its climatic impacts in the Eocene. Palaeogeography, Palaeoclimatology, Palaeoecology, v. 231, n. 1-2, pp. 9-28.

ICZN, 1999. International code of zoological nomenclature. 4a ed. London: The International Trust for Zoological Nomenclature/The Natural History Museum.

Ivany, L. C.; Van-Simaeyes, E. W.; Domack, E. W.; Samson, S. D. 2006. Evidence for an earliest Oligocene ice sheet on the Antarctic Peninsula. Geology, v. 34, pp. 337-380.

Jadwiszczak, P.; Mors, T. 2011. Aspects of diversity in early Antarctic penguins. Acta Palaeontologica Polonica, v. 56, n. 2, pp. 269-277.

Jones, R. W.; Charnock, M. A. 1985. "Morphogroups" of agglutinating foraminífera: Their life positions and feeding habits and potential applicability in (paleo)ecological studies. Revue de Paléobiologie, v. 4, pp. 311-320.

Jones, R. W. 1994. The Challenger foraminifera. London: Oxford University Press.

Kaminski, M. A.; Gradstein, F. M.; Berggren, W. A. 1988. Flysch-type agglutinated foraminiferal assemblages from Trinidad: Taxonomy, stratigraphy and paleobathymetry. Abh. Geol. B.-A., v. 41, pp. 155-227.

Kaminski, M. A. 2004. The year 2000 classification of the agglutinated foraminifera. In: Bubík, M.; Kaminski, M. A. (Eds.). Proceedings of the Sixth International Workshop on Agglutinated Foraminifera. Kraków: Grzybowski Foundation, pp. 237-255. 
Kaminski, M. A.; Gradstein, F. M. 2005. Atlas of Paleogene cosmopolitan deep-water agglutinated foraminifera. Kraków: The Grzybowski Foundation.

Kaminski, M. A.; Silye, L.; Kender, S. 2009. Miocene deep-water agglutinated foraminifera from the Lomonosov Ridge and the opening of the fram Strait. Micropaleontology, v. 55, n. 2-3, pp. 117-135.

Kazmierczak, J. 1976. Volvocacean nature of some Palaeozoic non-radiosphaerid calcispheres and parathuramminid "foraminifera". Acta Palaeontologica Polonica, v. 21, n. 3, pp. 245-258.

Kennett, J.; Srinivasan, M. S. 1983. Neogene planktonic foraminifera: A phylogenetic atlas. New York: Hutchinson Ross Publishing Company.

Kontorovich, A; Varlamov, A; Grazhdankin, D; Karlova, G; Klets, A; Kontorovich, V; Saraev, S; Terleev, A; Belyaev, S (2008). A section of Vendian in the east of West Siberian Plate (based on data from the Borehole Vostok 3). Russian Geology and Geophysics, v. 49, n. 2, pp. 932-939.

Krasheninnikov, V. A. 1973. Cretaceous benthonic foraminifera, Leg 20, Deep Sea Drilling Project. Initial Reports of the Deep Sea Drilling Project, v. 20, pp. 205-221.

Krasheninnikov, V. A. 1974. Upper Cretaceous benthonic agglutinated foraminifera, Leg 27, Deep Sea Drilling Project. Initial Reports of the Deep Sea Drilling Project, v. 27, pp. 631-661.

Krasheninnikov, V. A.; Pflaumann, U. 1977. Cretaceous agglutinated foraminifera of the Atlantic Ocean off West Africa (Leg 41, Deep Sea Drilling Project). Initial Reports, v. 41, pp. 565-580.

Krasheninnikov, V. A.; Basov, I. A. 1986. Late Mesozoic and Cenozoic stratigraphy and geological history of the South Atlantic high latitudes. Palaeogeography, Palaeoclimatology, Palaeoecology, v. 55, pp. 145-188.

Kurschner, W. M.; Kvacek, Z.; Dilcher, D. L. 2008. The impact of the Miocene atmospheric carbon dioxide fluctuations on climate and the evolution of terrestrial ecosystems. Proceedings of the National Academy of Sciences, v. 105, pp. 449-453.

Lagabrielle, Y.; Goddéris, Y.; Donnadieu Y, Malavieille J, Suarez M. 2009. The tectonic history of Drake Passage and its possible impacts on global climate. Earth Planetary Science Letters, v. 279, n. 3-4, pp. 197-211.

Lazarus, D.; Faust, K.; Popova-Goll, I. 2005. New species of prunoid radiolarians from the Antarctic Neogene. Journal of Micropaleontology, v. 24, pp. 97-121.

Lawver, L. A.; Gahagan, L. M. 2003. Evolution of Cenozoic seaways in the circum-Antarctic region. Palaeogeography, Palaeoclimatology, Palaeoecology, v. 198, pp. 11-37. 
Li, Q.; Radford, S. S.; Banner, F. T. 1992. Distribution of microperforate tenuitellid planktonic foraminifers in holes 747A and 749B, Kerguelen Plateau. Proceedings of the Ocean Drilling Program, Scientifc Results, V. 120, pp. 569-594.

Lipps, J. H.; Berger, W. H.; Buzas, M. A.; Douglas, R. G. Ross, C. A. 1979. Foraminiferal ecology and paleoecology. SEPM.

Lipps, J. H. 2011. What should we call the foraminifera? Journal of Foraminiferal Research, v. 41, n. 4, pp. 309-313.

Loeblich, A. R., Jr.; Tappan, H. 1964. Sarcodina, chiefly 'Thecamoebians' and Foraminiferida. In Moore, R. C. (Ed.). Treatise on Invertebrate Paleontology, Vol. C, Protista 2. Kansas: Geological Society of America/University of Kansas Press.

Loeblich, A. R. Jr.; Tappan, H. 1987. Foraminiferal genera and their classification. 2 vols. New York: Van Nostrand Reinhold.

Loeblich, A. R. Jr.; Tappan, H. 1992. Present status of foraminiferal classification: In: Takayanagi, Y; Saito, T. (Eds.). Studies in benthic foraminifera. Tokay: Tokay University Press, pp. 93-102.

Loeblich, A. R. Jr.; Tappan, H. 1994. Foraminifera of the Sahul Shelf and Timor Sea. Cushman Foundation for Foraminiferal Research, Special Publication, n. 31, 668 pp.

Long, D. J. 1992. Sharks from the La meseta Formation (Eocene), Seymour Island, Antarctic Peninsula. Journal of Vertebrate Paleontology, v. 12, n. 1, pp. 11-32.

Loubere, P.; Banonis, G.; Jakiel, R. 1988. Globocassidulina subglobosa (Brady): Environmental control of species abundance and specimen test size. Journal of Foraminiferal Research, v. 18, n. 1, pp 6-15.

Loubere, P.; Rayray, S. 2016. Benthic foraminiferal assemblage formation: Theory and observation for the European Arctic margin. Deep Sea Research I, v. 115, pp. 36-47.

Majewski W. 2005. Benthic foraminiferal communities: Distribution and ecology in Admiralty Bay, King George Island, West Antarctica. Polish Polar Research, v. 26, n. 3, pp. 159-214.

Majewski W. 2010. Benthic foraminifera from West Antarctic fiord environments: An overview. Polish Polar Research, v. 31, n. 1, pp. 61-82.

Majewski, W. 2013. Benthic foraminifera from the Pine Island and Ferrero bays, Amundsen Sea. Polar Polish Research, v. 34, n. 2, pp. 169-200.

Majewski W.; Gazdzicki A. 2014. Shallow water benthic foraminifera from the Polonez Cove Formation (Lower Oligocene) of King George Island, West Antarctica. Marine Micropaleontology, v. 111, pp. 1-14. 
Majewski, W.; Pawloski, J. 2010. Morphologic and molecular diversity of the foraminiferal genus Globocassidulina in Admiralty Bay, King George Island. Antarctic Science, v 22, n. 3, pp. 271281.

Malagnino, E. C.; Olivero, E. B.; Rinaldi, C. A.; Spikermann, Y. J. P. 1981. Aspectos geomórfologicos de la Isla Vicecomodoro Marambio, Antártida. In: VIII Congreso Geológico Argentino (San Luis), Actas II, pp. 883-896.

Marenssi, S. A. 2006. Eustatically controlled sedimentation recorded by Eocene strata of the James ross Basin, Antarctica: In: Francis, J. E.; Pirrie, D.; Crame, J. A. (Eds.). Cretaceous-Tertiary highlatitude paleoenvironments, James Ross Basin, Antarctica. London: Geological Society Special Publications, v. 258, pp. 125-133.

Marenssi, S. A.; Casadío, S.; Santillana, S. N. 2010. Record of Late Miocene glacial deposits on Isla Marambio (Seymour Island), Antarctic Peninsula. Antarctic Science, v. 22, n. 2, p. 193-198.

Marenssi, S. A.; Net, L. I.; Santillana, S. N. 2002. Provenance, environmental and paleogeographic controls on sandstone composition in an incised-valley system: the Eocene La Meseta Formation, Seymour Island, Antarctica. Sedimentary Geology, v. 150, pp. 301-321.

Marenssi, S. A.; Santillana, S.; Bauer, M. 2012. Estratigrafía, petrografía sedimentaria y procedencia de lar formaciones Sobral y Cross Valley (Paleoceno), Isla Marambio (Seymour), Antártica. Andean Geology, v. 39, n. 1., pp. 67-91.

Margulis, L. Chapman, M. J. 2010. Kingdoms and domains: An illustrated guide to the phyla of life on Earth. 4a ed. Amsterdam: Elsevier/Academic Press.

Martin, R. E. 1999. Taphonomy: A process approach. Cambridge: Cambridge University Press.

Martin, R. E.; Wehmiller, J. F.; Harris, M. S.; Liddell, D. R. 1996. Taphonomy of bivalves and foraminifera from Holocene tidal flat sediments, Bahia la Choya, Sonora, Mexico (Northern Gulf of California): Taphonomic grades and temporal resolution. Paleobiology, v. 22, n. 1, pp. 80-90.

Mayr, E. 1963. Animal species and evolution. Massachussets: Belknap Press.

Mayr, E.; Ashlock, P. D. 1991. Principles of systematic zoology. New York: McGraw-Hill.

McGowram, B. 2005. Biostratigraphy: Microfossils and geological time. Cambridge: Cambridge University Press.

McIlroy, D.; Green, O. R.; Brasier, M. D. 2001. Paleobiology and evolution of the earliest agglutinated foraminifera: Platysolenites, Spirosolenites and related forms. Lethaia, v. 34, pp. 1329

Mello-e-Souza, S. E.; Passos, R. F.; Burone, L.; Barbosa, V. P.; Mahiques, M. M.; Alves, D. P. V.; Koutsoukos, E. A. M. 2012. Foraminíferos planctônicos e bentônicos da plataform e talude 
continental do Atlântico Sudoeste, entre $19^{\circ}-33^{\circ}$ S. São Paulo: Editora da Universidade de São Paulo.

Miller, K. G.; Gradstein, F. M.; Berggren, W. A. 1982. Late Cretaceous to Early Tertiary agglutinated benthic foraminifera in the Labrador Sea. Micropaleontology, v. 28, pp. 1-30.

Miller, W., III. 1995, Examples of Mesozoic and Cenozoic Bathysiphon (Foraminiferida) from the Pacific Rim and the taxonomic status of Terebellina Ulrich, 1904. Journal of Paleontology, v. 69, pp. 624-634.

Milner, G. J. 1997. Paleogene foraminiferal studies in the Western Pacific. Part A. Paleocene foraminiferal biostratigraphy of the Papuan Basin and adjoining areas, Papua New Guinea; Part B. Middle Eocene to early Oligocene foraminifers from the Izu-Bonin fore-arc, Hole 786A. Tese de doutorado, University of Western Australia, $302 \mathrm{pp}$

Mikhalevich, V. I. 2004. On the heterogeneity of the former Textulariina (Foraminifera). In: Bubik,M; Kaminski, M. A. (Eds.). Proceedings of the Sixth International Workshop on Agglutinated Foraminífera. Kraków: Grzybowski Foundation, pp. 317-349.

Moodley, L.; Van der Zwaan, G. J.; Herman, P. M. J. Kempers, L.; Van Breugel, P. 1997. Differential response of benthic meiofauna to anoxia with special reference to Foraminifera (Protista; Sarcodina). Marine Ecology Progress Series, v. 158, pp. 131-163.

Mosbrugger, V.; Torsten, U.; Dilcher, D. L. 2005. Cenozoic continental climatic evolution of central Europe. PNAS, v. 102, n. 42, pp. 14964-14969.

Muftah, A. M. 1995. Agglutinated foraminifera from the Danian sediments of the northeastern Sirte Basin. In: Kaminski, M. A.; Geroch, S.; Kaminski, M. A. (Eds.). 1995. Proceedings of the Fourth International Workshop on Agglutinated Foraminifera. Kraków: Grzybowski Foundation, pp. 173-180.

Murray, J. W. 1991. Ecology and paleoecology of benthic foraminifera. Cambridge: Logman Group.

Naeher, S.; Geraga, M.; Papatheodorou, G.; Ferentinos, G.; Kaberi, H.; Schubert, C. J. 2012. Environmental variations in a semi-enclosed embayment (Amvrakikos Gulf, Greece): Reconstructions bsed on benthic foraminifera abundance and lipid biomarker pattern. Biogeosciences, v. 9, pp. 5081-5094.

Nelson, D. M.; Tréguer, P.; Brzezinski, M. A.; Leynaert, A.; Quéguiner, B. 1995. Production and dissolution of biogenic silica in the ocean: Revised global estimates, comparison with regional data and relationship to biogenic sedimentation. Global Biogeochemical Cycle, v. 9, n. 3, pp. 359372.

Nelson, P. H. 1975. The James Ross island Volcanic Group of north-east Graham Land. British Antarctic Survey, Scientific Report, v. 54, pp. 1-62. 
Pagani, M.; Zachos, J. C.; Freeman, K. H.; Tipple, B.; Bohaty, S. 2005. Marked decline in atmospheric carbon dioxide concentrations during the Paleogene. Science, v. 309, p. 600-603.

Patterson, R. T. 1993. Late Quaternary benthic foraminiferal biofacies and paleoceanography of Queen Charlotte Sound and southern Hecate Strait, British Columbia. Journal of Foraminiferal Research, v. 23, n. 1, pp. 1-18.

Patterson, R. T.; Richardson, R. H. 1988. Eight new genera of unilocular foraminifera. Transactions of the American Microscopical Society, v. 107, n. 3, pp. 240-258.

Patterson, R. T.; Guilbault J. P.; Clague, J. J. 1999. Taphonomy of tidal marsh foraminifera: Implications of surface sample thickness for high-resolution sea-level studies. Palaeogeography, Palaeoclimatology, Palaeoecology, v. 149, pp. 199-211.

Pawlowski, J.; Holzmann, M.; Tyszka, J. 2013. New supraordinal classification of Foraminifera: Molecules meet morphology. Marine Micropaleontology, v. 100, pp. 1-10.

Pearson, P. N. 2010. Increased atmospheric $\mathrm{CO}_{2}$ during the Middle Eocene. Science, v. 330, n. 6005, pp. 763-764.

Pearson, P. N.; Foster, G. L.; Wade, B. S. 2009. Atmospheric carbon dioxide through the EoceneOligocene climate transition. Nature, v. 461, pp. 1110-1113.

Petrushevskaya, M. G.; Kozlova, G. E. 1979. Opisanie rodov i vidov radiolyarii. In: Strelkov, A. A.; Petrushevskaya, M. G. (Eds.). Issledovaniya fauny morei. Istoziya mikorplanktona norvezhskogo morya (po materialom glubokovodnogo bureniya). Leningrad: Nauka Academy of Sciences of the USSR, pp. 86-157.

Pflum, C. E. 1966. The distribution of foraminifera in the Eastern Ross Sea, Amundsen Sea, and Bellingshausen Sea, Antarctica. Bulletins of American Paleontology, v. 50, n. 226, pp. 151-209.

Phleger, F. B. 1960. Ecology and distribution of recent foraminifera. Baltimore: The John Hopkins Press.

Phleger, F. B.; Soutar, A. 1973, Production of benthic foraminifera in three east Pacific oxygen minima. Micropaleontology, v. 19, pp. 110-115.

Pirrie, D.; Duane, A. M.; Riding, J. B. 1992. Jurassic-Tertiary stratigraphy and palynology of the James Ross Basin: Review and introduction. Antarctic Science, v. 4, n. 3, pp. 259-266.

Pirrie, D.; Crame, J. A.; Lomas, S. A.; Riding, J. B. 1997a. Late Cretaceous stratigraphy of the Admiralty Sound region, James ross Basin, Antarctica. Cretaceous Research, v. 18, pp. 109-137.

Pirrie, D.; Crame, J. A.; Riding, J. B.; Butcher, A. R.; Taylor, P. D. 1997b. Miocene glaciomarine sedimentation in the northern Antarctic Peninsula region: The stratigraphy and sedimentology of the Hobbs Glacier Formation, James Ross Island. Geological Magazine, v. 136, n. 6, pp. 745-762. 
Pokorny, V. 1963. Principles of zoological micropaleontology. New York: Pergamon Press.

Porebski, S. Shel-valley compound fill produced by fault subsidence and eustatic sea-level changes, Eocene La Meseta Formation, Seymour Island, Antarctica. Geology, v. 28, n. 2, pp. 147150 .

Postuma, J. A. 1971. Manual of planktonic foraminifera. Amsterdam: Elsevier.

Pound, M.; Haywood, A. M.; Salzman, U.; Riding, J. B. 2012. Global vegetation dynamics and latitudinal temperature gradients during the Mid to Late Miocene (15.97-5.33 Ma). Earth-Science Reviews, v. 112, n. 1-2, pp. 1-22.

Prothero, D. R. 2009. Greenhouse of the dinosaurs: Evolution, extinction, and the future of our planet. New York: Columbia University Press.

Prothero, D. R. 2013. Bringing fossils to life: An introduction to paleobiology. 3a ed. New York: Columbia University Press.

Quaglio, F. Taxonomia de invertebrados fósseis (Oligoceno-Mioceno) da Ilha Rei George (Antártica Ocidental) e paleobiogeografia dos bivalvia cenozoicos da Antártica. Tese de doutorado, Instituto de Geociências da Universidade de São Paulo

Renaudie, J. 2012. A synthesis of Antarctic Neogene radiolarians: Taxonomy, macroevolution und biostratigraphy. Tese de doutorado, Mathematisch-Naturwissenschaftlichen Fakultät I der Humboldt, Universität zu Berlin.

Renaudie, J.; Lazarus, D. B. 2012. New species of Neogene radiolarians from the Southern Ocean. Part 4. Journal of Micropalaeontology, v. 35, pp. 26-53,

Retelle, M. W.; Weddle, T. K. 2011. Deglaciation and relative sea-level chronology, Casco Bay Lowland and lower Androscoggin River Valley, Maine. In: Weddle, T. K.; Retelle, M.W. (Eds.). Deglaciation history and relative sea-level changes, northern New England. Geological Society of America, pp. 193-216.

Reuss, A. E. 1861. Entwurf einer systematischen zummenstellung der foraminiferen. Sitzungsberichte der K. Akademie der Wissenschaften, Wien, v. 44, pp. 354-396.

Revets, S. A. 2005. A key to the unilocular hyaline foraminifera. Journal of Micropalaeontology, v. 24, pp. 145-158.

Richardson, S. L.; Clayshuttle, R. N. 2003. An annotated checklist of foraminifera of Guam. Micronesica, v. 35-36, pp. 38-53.

Ricklefs, R. E. 2010. A economia da natureza. 6a ed. Rio de Janeiro: Guanabara Koogan. 
Riding, J. B.; Crame, J. A.; Dettmann, M. E.; Cantrill, D. J. 1998. The age of the base of the Gustav Group in the James Ross Basin, Antarctica. Cretaceous Research, v. 19, pp. 87-105.

Rigby, J. K. 1987. Phylum Porifera. In: Boardman, R. S.; Cheetham, A. H.; Rowell, A. J. (Eds.). Fossil invertebrates. Oxford: Blackwell Scientific Press, pp. 116-139.

Riedel, W. R. 1971. Systematic classification of polycistine radiolaria. In: Funnell, M.; Riedel, W. R. (Eds.). The micropalaeontology of oceans. Cambridge: Cambridge University Press, pp. -.

Robinson, M.; Dowsett, H. J.; Chandler, M. A. 2008. Pliocene role in assessing future climate impacts. EOS, Transactions, American Geophysical Union, v. 89, n. 49, pp. 501-502.

Rocha-Campos, A. C.; Kuchenbecker, M.; Duleba, W.; Santos, P. R.; Canile, F. M. 2017. A (tidalmarine) boulder pavement in the Late Cenozoic of Seymour Island, west Antarctica: Contribution to the palaeogeographical and palaeoclimatic evolution of west Antarctica. Antarctic Science, v. 29 , n. 5, pp. 1-5.

Rodrigues, A. R. 2008. Estudo das associações de foraminíferos bentônicos recentes na Baía do Almirantado (Ilha Rei George, Antártica) durante três verões austrais consecutivos. Tese de doutorado, Instituto Oceanográfico da Universidade de São Paulo.

Rögl, F.; Bolli, H. M. 1973. Holocene to Pleistocene planktonic foraminifera of Leg 15, Site 147 (Cariaco Basin (Trench), Caribbean Sea) and their climatic interpretation. Initial Reports of the Deep Sea Drilling Project, v. 15, pp. 553-616.

Rowley, D. B. 2002. Rate of plate creation and destruction: 180 Ma to present. Geological Society of America Bulletin, v. 114, pp. 927-933.

Ruffinatti, C. P.; Hoyos, C. L. 1981. Foraminiferos recientes de algunos puntos del Caribe frente a las costas colombianas. Caldasia, v. 13, n. 62, pp. 257-289.

Ruppert, E. E.; Fox, R. S.; Barnes, R. D. 2005. Zoologia dos invertrebados: Uma abordagem functional-evolutiva. 7a ed. São Paulo: Roca.

Sabbatini, A.; Morigi, C.; Ravaioli, M.; Negri, A. 2004. Abyssal benthic foraminifera in the Polar Front region (Pacific sector): Faunal composition, standing stock and size structure. Chemistry and Ecology, v. 20, pp. 117-129.

Sadler, P. M. 1988. Geometry and stratification of the uppermost Cretaceous and Paleogene units on Seymour Island, northern Antarctic Peninsula. Geological Society of America Memoirs, v. 169, pp. 303-320.

Santos, P. R. 1996. Condicionamento geológico da sedimentação glacial: O Neopaleozoico da Bacia do Paraná e o Terciário da Antártica. São Paulo. Tese de livre-docência, Instituto de Geociências da Universidade de São Paulo. 
Scher, H. W.; Martin, E. E. 2006. Timing and climatic consequences of the opening of Drake Passage. Science, v. 312, pp. 428-430.

Schiebel, R.; Hemleben, C. 2017. Planktic foraminifers in the modern ocean. Springer.

Schröder-Adams, C. J. 1990. High latitude agglutinated foraminifera: Prydz Bay (Antarctica) vs. Lancaster Sound (Canadian Arctic). In: Hemleben, C.; Kaminski, M. A.; Kuhnt, W.; Scott, D. B. (Eds.). Paleoecology, Biostratigraphy, Paleoceanography and Taxonomy of agglutinated foraminifera. New York: Kluwer Academic Press, pp. 315-343.

Schröder-Adams, C. J.; McNeil, D. H. 1994a. New paleoenvironmentally important species of agglutinated foraminifera from the Oligocene and Miocene of the Beaufort Sea, Arctic Canada. Journal of Foraminiferal Research, v. 24, pp. 178-190.

Schröder-Adams, C. J.; McNeil, D. H. 1994b. Oligocene to Miocene agglutinated foraminifera in deltaic and deep-water facies of the Beaufort-MacKenzie Basin. Geological Survey of Canada Bulletin, v. 477, 67 pp.

Schultze, M. J. S. 1854. Über den organismus der Polythalamien (Foraminiferen), nebst Bemerkungen über die Rhizopoden im allgemeinen. Leipzig, Ingelmann.

Scott, D. B.; Medioli, F.; Braund, R. 2003. Foraminifera from the Cambrian of Nova Scotia: The oldest multichambered foraminifera. Micropaleontology, v. 49, n. 2, pp. 109-126

Sen Gupta, B. K. 1999. Foraminifera in marginal marine environments. In: Sen Gupta, B. K. (Ed.). Modern foraminifera. Dordrecht: Kluwer Academic Publishers, pp. 141-159.

Sen Gupta, B. K.; Smith, L. E.; Machain Castillo, M. L. 2009. Foraminifera of the Gulf of Mexico. In: Felder, D.L.; Camp, D. K. (Eds.). Gulf of Mexico: Origins, waters, and biota. Texas: A\&M Press, pp. 87-129.

Shevenell, A. E.; Kennett, J. P.; Lea, D. W. 2008. Middle Miocene ice sheet dynamics, deep-sea temperatures, and carbon cycling: A Southern Ocean perspective. Geochemistry, Geophysics, Geosystems, v. 9, n. 2, pp. 1-14.

Sinha, H.; Nigam, R.; Khare, N. Ingole, B. S.; Ghosh, D. N. 2000. Preliminary report on the study of benthic foraminiferal content in the marine sediments of Queen Maud Land Shelf, Lazarev Sea, Antarctica. ONGC Bulletin, v. 37, n. 2, pp. 49-53.

Smellie, J. L.; McArthur, J. M.; Mcintosh. W. C.; Esser, R. 2006. Late Neogene interglacial events in the James Ross Island region, northern Antarctic Peninsula, dated by Ar/Ar and Sr-isotope stratigraphy. Palaeogeography, Palaeoclimatology, Palaeoecology, v. 242, n. 3-4, pp. 169-187

Sorlien, C. C.; Luyendyk, B. P.; Wilson, D. S.; Decesari, R. C.; Bartek, L. R.; Diebold, J. B. 2007. Oligocene development of the West Antarctic Ice Sheet recorded in eastern Ross Sea strata. Geology, v. 35, n. 5, pp. 467-470. 
Speelman, E. N.; Kempen, M. M. L.; Barke, J.; Brinkhuis, H.; Reichart, G. J.; Smolders, A. J. P.; Roelofs, J. G. M.; Sangiori, F.; Leeuw, J. W.; Lotter, A. F.; Sinninghe Damsté, J. S. 2009. The Eocene Arctic Azolla bloom: Environmental conditions, productivity and carbon drawdown. Geobiology, v. 7, pp. 155-170.

Spezzaferri, S.; Rögl, F.; Ćorić, S.; Hohenegger, J. 2004. Paleoenvironmental changes and agglutinated foraminifera across the Karpatian/Badenian (Early/ Middle Miocene) boundary in the Styrian Basin (Austria, Central Paratethys). In: Bubík, M.; Kaminski, M. A. (Eds.). Proceedings of the Sixth International Workshop on Agglutinated Foraminifera. Kraków: Grzybowski Foundation Special Publication, pp. 423-459.

Stilwell, J. D.; 2002. Geological exploration of Cockburn Island, Antarctic Peninsula. Polish Polar Research, v. 23, n. 1, pp. 1-27.

Stilwell, J. D.; Zinsmeister, W. J. 1987. Late Cretaceous fossils from Cockburn Island collected during the 1986-1987 expedition to the Antarctic Peninsula. Antarctic Journal of the United States, v. 22, n. 5, pp. 5-6.

Stilwell, J. D.; Zinsmeister, W. J. (Eds.). 1992. Molluscan systematics and biostratigraphy: Lower tertiary La Meseta Formation, Seymour Island, Antarctic Peninsula. Washington: American Geophysical Union.

Stoermer, E. F.; Julius, M. L. 2003. Centric Diatoms. In: Wehr, J. D.; Sheath, R. G. (Eds.). Freshwater algae of North America: Ecology and classification. Amsterdam: Academic Press, pp. 559-594.

Strong, C. P. 1984. Cretaceous-Tertiary boundary, Mid-Waipara River section, North Canterbury, New Zealand. New Zealand Journal of Geology and Geophysics, v. 27, pp. 231-234.

Strong, C. P.; Webb, P. N. 2000. Oligocene and Miocene foraminifera from CRP-2/2A, Victoria Land Basin, Antarctica. Terra Antarctica, v. 7, n. 4, pp. 461-472.

Svojtka, M.; Nyvlt, D.; Murakami, M.; Vavrova, Jitka, J.; Filip, J.; Mixa, P. 2009. Provenance and pot-deposicional low-temperature evolution of the James Ross Basin sedimentary rocks (Antarctic Peninsula) based on fission track analysis. Antarctic Science, v. 26, n. 6, pp. 593-607.

Szarek, R.; Kitazato, H.; Nomaki, H.; Shimanaga, M. 2005. Deep-sea benthic foraminiferal assemblages from the dysoxic Sulu Sea: Modern analogue for the mid-Cretaceous warm dysoxic ocean. Preliminary results. Frontier Research on Earth Evolution, v. 2, pp. 1-5.

Székely, S. F.; Filipescu, S. 2015. Taxonomic record of the Oligocene benthic foraminifera from the Vima Formation (Transylvanian Basin, românia). Acta Palaeontologica Romaniae, v. 11, n. 1, pp. 25-62. 
Tambussi, C. P.; Noriega, J. I.; Gazdzicki, A.; Tatur, A.; Reguero, M. A.; Vizcaino, S. F. 1994. Ratite Bird from the Paleogene La Meseta Formation, Seymour Island, Antarctica. Polish Polar Research, v. 15, n. 1-2, pp. 15-20.

Thomas, E. 1989. Development of Cenozoic deep-sea benthic foraminiferal faunas in Antarctic waters. Geological Society London Special Publication, v. 47, pp. 283-296.

Thomas, E., 1998, The biogeography of the Late Paleocene benthic foraminiferal extinction, In: Aubry, M. P.; Lucas, S.; Berggren, W. A. (Eds.). Late Paleocene-early Eocene Biotic and Climatic Events in the Marine and Terrestrial Records. New York: Columbia University Press, p. 214-243

Toggweiler, J. R.; Bjornsson, H. 2000. Drake Passage and paleoclimate. Journal of Quaternary Science, v. 15, n. 4, pp. 319-328.

Trautman, T. A. 1976. Stratigraphy and petrology of Tertiary clastic sediments, Seymour Island. Dissertação de mestrado. The Ohio State University.

Troedson, A. L.; Smellie, J. L. 2002. The Polonez Cove Formation of King George Island, Antarctica: Stratigraphy, facies and implications for mid-Cenozoic cryosphere development. Sedimentology, v. 49, pp. 277-301.

Uchman, A.; Gazdzicki, A. 2006. New trace fossils from the La Meseta Formation (Eocene) of Seymour Island, Antarctica. Polish Polar Research, v. 27, n. 2, pp. 153-170.

Uriz, M. J. 2002. Family Geodiidae Gray, 1867. In: Hooper, J. N. A.; Soest, R. W. M. (Eds.). 2013. Systema Porifera: A guide to the classification of sponges. V. 1. Heilderberg: Springuer, pp. 134140.

Uriz, M. J. 2006. Mineral skeletogenesis in sponges. Canadian Journal of Zoology, v. 84, pp. 322356.

Vénec-Peyré, M. T. 2005. Les planches inédites de foraminifères d'Alcide d'Orbigny: À l'aube de la micropaléontologie. Paris: Muséum National d'Histoire Narurelle.

Versteegh, G. J. M.; Servais, T.; Streng, M.; Munneckes, A.; Vachard, D. 2009. A discussion and proposal concerning the use of the term calcispheres. Palaeontology, v. 52, n. 2, pp. 343-348.

Waddell, L. M.; Moore, T. C. 2008. Salinity of the Eocene Arctic Ocean from oxygen isotope analysis of fish bone carbonate. Paleoceanography, v. 23, n. 1, pp. 1-14.

Webb, P. N. 1971. New Zealand Late Cretaceous (Haumurian) foraminifera and stratigraphy: New Zealand Journal of Geology and Geophysics, v. 14, pp. 795-828.

Webb, P. N. 1973. Paleocene foraminífera from Wangaloa and Dunedin. New Zealand Journal of Geology and Geophysics, v. 16, pp. 109-157. 
Webb, P. N. 1974. Micropaleontology, paleoecology and correlation of the Pecten Gravels, Wright Valley, antarctica, and description of Trochoelphidella onyxi n. gen., n. sp. Journal of Foraminiferal Research, v. 4, pp. 84-199.

Webb, P. N. 1975. Paleocene foraminifera from DSDP Site 283, South Tasman Basin. Initial Reports of the Deep Sea Drilling Project, v. 29, pp. 833-843.

Webb, P. N. 1988. Upper Oligocene-Holocene foraminifera of the Ross Sea region. Revue de Paléobiologie, Special volume 2, n. 2, pp. 589-603.

Welton, B. J.; Zinsmeister, w. J. 1980. Eocene Neoselachians from the La Meseta Formation, Seymour Island, Antarctic Peninsula. Contributions in Science, n. 329, pp. 1-10.

Wendler, J. E.; Wendler, I.; Huber, B. T. MacLeod, K. G. 2010. What are calcispheres? Pristine specimens from the Tanzania drilling projet provide unprecedented insight into an enigmatic microfossil group. In: GSA Denver Annual Meeting. Abstracts with programs, p. 131.

Wetzel, F.; Souza, G. F.; Reynolds, B. C. 2014. What controls silicon isotope fractionation during dissolution of diatom opal? Geochimica et Cosmochimica Acta, v. 131, pp. 128-137.

You, Y.; Huber, M.; Muller, R. D.; Poulsen, C. J.; Ribbe, J. 2009. Simulation of the Middle Miocene Climate Optimum. Geophysical Research Letters, v. 36, p. 1-5.

Zachos, J. C.; Berggren, W. A.; Aubry, M. P.; Mackensen, A. 1992. Isotope and trace element geochemistry of Eocene and Oligocene foraminifers from Site 748, Kerguelen Plateau. In: Wise, S. W.; Schlich, R. (Eds.). Proceedings of the Ocean Drilling Program, Scientific Results, v. 120, pp. 839-854.

Zachos, J. C.; Pagani, M.; Sloan, L.; Thomas, E.; Billups, K. 2001. Trends, rhythms, and aberrations in global climate 65 Ma to present. Science, v. 292, pp. 686-693.

Zachos, J. C.; Dickens, G. R.; Zeebe, R. E. 2008. Na Early Cenozoic perspective on greenhouse warming and carbono-cycle dynamics. Nature, v. 451, pp. 279-283.

Zhang, Y. G.; Pagani, M.; Liu, Z.; Bohaty, M. DeConto, R. 2013. A 40-million-year history of atmospheric $\mathrm{CO}_{2}$. Philosophical Transactions of the Royal Society A, v. 371, pp. 1-20.

Zinsmeister, W. J. 1984. Late Eocene bivalves (Mollusca) from the La Meseta Formation, collected during the 1974-1975 joint Argentine-American expedition to Seymour Island, Antarctic Peninsula. Journal of Paleontology, v. 58, n. 6, pp. 1497-1527.

Zinsmeister, W. J.; DeVries, T. J. 1982. Observations on the stratigraphy of the Lower Tertiary Seymour Island Group, Seymour Island, Antarctic Peninsula. Antarctic Journal of the United States, v. 17, pp. 71-72. 
